\title{
The Complexity of Stratification Computation
}

\author{
E. Rannou \\ Département de Mathématiques, Université de Bretagne Occidentale, \\ 29285 Brest Cedex, France \\ Eric.Rannou@univ-brest.fr
}

\begin{abstract}
This paper investigates the complexity of stratification computation for semialgebraic sets. An upper bound for the computation of canonical stratifications is given for a wide class of stratifying conditions called here admissible. For such conditions, the stratifying process is at most doubly exponential in the depth of the stratification. Usual conditions of regularity like Whitney conditions (a) and (b) or Bekka condition (C) are admissible. A useful criterion and tools are given in order to prove easily other admissibilities.
\end{abstract}

\section{Introduction}

The basic fact in the theory of stratifications is that it is always possible to decompose an algebraic set or a semialgebraic set into a union of connected smooth manifolds of various dimensions, called strata. Roughly speaking, a stratification is such a decomposition.

The first idea in order to construct a stratification is to construct the singular locus of the algebraic set, which is an algebraic set of lower dimension. Then again the singular locus of the singular locus is constructed, and so on. The depth of the stratification is the number of times this construction has to be iterated. Unfortunately, this construction does not permit a good control of the topology of the set along strata, and it is necessary to introduce regularity conditions on stratifications (e.g., conditions on the limits of the tangent spaces).

In order to construct stratifications satisfying certain regularity conditions, it is possible to do as follows: for every pair of strata $X$ and $Y$ already computed with $\operatorname{dim}(X) \leq$ $\operatorname{dim}(Y)$ for which the closure of $Y$ contains $X$, the set of bad points of $X$ is constructed as the set of points at which the regularity condition is not satisfied. In several important examples, the dimension of the set of bad points is always strictly smaller than the dimension of $X$, which means that the construction will terminate. Regularity conditions with such properties are called stratifying conditions. The depth of the stratification is the number of iterations needed by the construction to be ended. 
Here we are going to study the complexity of an algorithm that constructs such stratifications for general semialgebraic sets and prove that it is at most doubly exponential in the depth of the stratification. Our result is based on the following ideas. We will note that a wide class of stratifying conditions is admissible, in the way that the description of the set of bad points on a stratum is singly exponential in the number of variables. This observation relies on recent results about the single exponential complexity of various algorithms in real algebraic geometry: elimination of quantifiers when the number of alternations is fixed, computation of connected components. To iterate this construction leads to an algorithm which has double exponential complexity in the depth of the stratification.

In 1989, Canny [C] used Whitney stratifications to estimate the complexity of the construction of a roadmap for a semialgebraic set. This has stimulated interest in the problem of the complexity of algorithms that compute these stratifications. Using the cylindrical algebraic decomposition algorithm of Collins, it is quite easy to get a bound doubly exponential in the number of variables. In 1991, Vorobjov [V] proved that the sequential complexity is singly exponential in the number of variables, for a wide class of semialgebraic sets.

The paper is organized as follows:

In the first section, we review some definitions and properties of semialgebraic sets and their stratifications. We define stratifying conditions, give examples, and describe the method used to construct stratifications satisfying a given stratifying condition.

In the second section, we recall some known results about the complexity of basic algorithms in semialgebraic geometry. Then we give some applications to the computation of the dimension and the singular locus, used later, in the next section.

In the third section, we recall some algorithmic results and construct some algorithmic tools used during the stratification computations. The most important of these tools is the $C^{k}$-locus algorithm.

In the fourth section, we study the complexity of an algorithm that stratifies semialgebraic sets. We give the definition of an admissible stratifying condition. We get a general theorem on the complexity of an algorithm which constructs a stratification for an admissible condition. This algorithm is doubly exponential in the depth of the stratification. Then we prove that several important cases of stratifying conditions, for example, the conditions (a) and (b) of Whitney, are admissible stratifying conditions. Then various specific results follow easily from the general theorem.

The last section is devoted to similar questions over the complex numbers.

\section{Stratifications: An Outline of Definitions and Results}

\subsection{Semialgebraic and Constructible Sets}

Let $\mathbf{K}$ be the field of real numbers $\mathbf{R}$ or the field of complex numbers $\mathbf{C}$.

Let $V$ be a subset of $\mathbf{K}^{n}$. Let $k$ be an integer number.

$* \bar{V}$ denotes the Euclidean closure of the subset $V$ of $\mathbf{K}^{n}$.

* $V_{\text {reg } k}$ denotes the set of all the points of $V$ which are $C^{k}$-smooth in $V, V_{\text {sing } k}$ is the set of all other points of $V$. 
* $V_{\text {reg }}$ denotes the set of all the points of $V$ which are $C^{\infty}$-smooth in $V, V_{\text {sing }}$ is the set of all other points of $V$.

* For each integer number $k$ between 0 and $n$, let $\mathbf{G}_{n, k}(\mathbf{K})$ be the Grassmannian of $k$-dimension vector subspace of $\mathbf{K}^{n}$.

Definition 1. Semialgebraic sets of $\mathbf{R}^{n}$ form the smallest family of subsets of $\mathbf{R}^{n}$ containing all the sets $\left\{x \in \mathbf{R}^{n}: P(x)>0\right\}$ where $P$ is a polynomial of $\mathbf{R}\left[X_{1}, \ldots, X_{n}\right]$ and which is closed with respect to the set-theoretic operations of finite union, finite intersection, and complementation.

All algorithms for the stratification computation need a convenient representation of sets. Usually, first-order formulas of ordered fields with parameters in $\mathbf{R}$ are used to define semialgebraic sets of $\mathbf{R}^{n}$ and constructible sets of $\mathbf{C}^{n}$. Then, to compute with such sets by algorithms is to compute with polynomials and logical connectors.

Definition 2. A formula is a first-order formula of the language of ordered fields with parameters in $\mathbf{R}$ if and only if it is built in a finite number of steps with polynomials with real coefficients, with logical connectors (or, and, not) and with quantifiers $(\exists, \forall)$ only on elements of the field $\mathbf{R}$.

In order to abbreviate, such a formula will be called a formula of $\mathbf{R}$ in this paper.

Definition 3. Constructible sets of $\mathbf{C}^{n}$ form the smallest family of subsets of $\mathbf{C}^{n}$ containing all the zero sets $\left\{x \in \mathbf{C}^{n}: P(x)=0\right\}$ where $P$ is a polynomial of $\mathbf{C}\left[X_{1}, \ldots, X_{n}\right]$ and which is closed with respect to the set-theoretic operations of finite union, finite intersection and complementation.

Proposition 1 [BCR, p. 47]. Let $V \subset \mathbf{R}^{n}$ be a semialgebraic set. Then, the set $V$ is the finite union of semialgebraic sets $V_{i}$ semialgebraically homeomorphic to $] 0,1\left[{ }^{d_{i}}\right.$ for some $d_{i}$ (where $] 0,1\left[{ }^{0}\right.$ denotes $\{0\}$ ).

Definition 4 [BCR, p. 47]. Let $V \subset \mathbf{R}^{n}$ be a finite union of semialgebraic sets $V_{i}$ semialgebraically homeomorphic to $] 0,1\left[{ }^{d_{i}}\right.$ for some $d_{i}$ (where $] 0,1\left[{ }^{0}\right.$ denotes $\{0\}$ ). Let $x$ be a point of $V$. The local dimension, $\operatorname{dim}_{x} V$, of $V$ at $x$ is given by $\operatorname{dim}_{x} V=\max _{x \in \bar{V}_{i}} d_{i}$.

\subsection{Stratification}

Definition 5. Let $V$ be a semialgebraic set of $\mathbf{R}^{n}$ (resp., a constructible set of $\mathbf{C}^{n}$ ). Let $k$ be a positive integer number.

A $C^{k}$-stratification $\mathcal{S}$ of $V$ is a finite decomposition in connected semialgebraic (resp., constructible) $C^{k}$-smooth sets of $V$ such that: the Euclidean closure of each element of $\mathcal{S}$ is a union of elements of $\mathcal{S}$ (boundary condition). The elements of $\mathcal{S}$ are called strata of the $C^{k}$-stratification. If all strata of $\mathcal{S}$ are $C^{\infty}$-manifolds, then $\mathcal{S}$ is called a stratification of the set $V$. 
Remarks. (i) $C^{k}$-stratifications appear here to avoid the computation's difficulty of the singular locus of a semialgebraic set of $\mathbf{R}^{n}$. Mather [M, p. 209] previously used this extension of the definition of stratification. Algorithms for $C^{k}$-locus determination, for $k$ fixed, can be built with reasonable complexities (i.e., singly exponential in the number of variables $n$ (Propositions 2 and 3)). If $k$ is large enough, $C^{k}$ implies $C^{\infty}$. But the known bound on $k$ does not allow us to obtain the expected sequential complexity. For constructible sets of $\mathbf{C}^{n}, C^{1}$ and $C^{\infty}$ are equivalent. Thus, only stratifications are considered in the complex case.

(ii) The aim is to construct explicitly stratifications of semialgebraic sets of $\mathbf{R}^{n}$ or constructible sets of $\mathbf{C}^{n}$. All computed stratifications are finite. The classical definition of stratification is formulated for more general sets and allows locally finite partitions.

(iii) The boundary condition can be expressed as follows:

$$
\text { for all strata } X \text { and } Y \text { of } \mathcal{S}, \quad \bar{X} \cap Y \neq \emptyset \quad \Longleftrightarrow \quad Y \subset \bar{X} \text {. }
$$

* Stratification of Maps

Definition 6. Let $f$ be a semialgebraic map (i.e., the graph of $f$ is semialgebraic) between two semialgebraic sets $V$ of $\mathbf{R}^{n}$ and $W$ of $\mathbf{R}^{p}$. Let $k$ be a positive integer number or the infinity.

A $C^{k}$-stratification of $f$ is a pair $\left(\mathcal{S}, \mathcal{S}^{\prime}\right)$ where $\mathcal{S}$ is a $C^{k}$-stratification of $V$ and $\mathcal{S}^{\prime}$ is a $C^{k}$-stratification of $W$, such that:

(i) $f$ maps each stratum of $\mathcal{S}$ in a stratum of $\mathcal{S}^{\prime}$; and

(ii) let $X$ be a stratum of $\mathcal{S}$, which is sent by $f$ in the stratum $Y$ of $\mathcal{S}^{\prime}$, then $\left.f\right|_{X}$ : $X \rightarrow Y$ is submersive (i.e., for each point $x$ of $X,\left(\left.d f\right|_{X}\right)_{x}$ is surjective where

$$
\begin{aligned}
\left(\left.d f\right|_{X}\right)_{x}: & T_{x}^{X} \\
\left(t_{1}, \ldots, t_{n}\right) & \left.\longmapsto\left(\frac{\partial f_{i}}{\partial x_{j}}(x)\right)_{i, j}^{Y} \cdot{ }^{t}\left(t_{1}, \ldots, t_{n}\right)\right) .
\end{aligned}
$$

The map $f:(V, \mathcal{S}) \longrightarrow\left(W, \mathcal{S}^{\prime}\right)$ is called a $C^{k}$-stratified map.

\subsection{Stratifying Condition: Definitions}

Definition 7. Let $k$ be an integer number or the infinity. A stratifying condition of order $k$ on $\mathbf{R}$ (resp., on $\mathbf{C}$ ) is a condition:

(i) on the triplets $(X, Y, y)$ where $X$ and $Y$ are two disjoint semialgebraic sets of $\mathbf{R}^{n}$ (resp., constructible sets of $\mathbf{C}^{n}$ ) and $y$ is a point of $Y$ such that:

- $Y \subset \bar{X}$;

- $X$ is a $C^{k}$-variety; and

- $y$ is $C^{k}$-smooth point of $Y$; 
(ii) satisfying the three following conditions:

* If the condition holds at $(X, Y, y)$ and if $Z$ is a semialgebraic (resp., constructible) $C^{k}$-smooth subset of $Y$ and containing the point $y$, then the condition holds at $(X, Z, y)$.

* For all triplets $(X, Y, y)$ where $X$ and $Y$ are two disjoint $C^{k}$-smooth semialgebraic sets of $\mathbf{R}^{n}$ (resp., $C^{k}$-smooth constructible sets of $\mathbf{C}^{n}$ ) such that $Y \subset \bar{X}$ and $y$ is a point of $Y$ and for all open semialgebraic (resp., constructible) subsets $Z$ of $Y$ containing the point $y$, the condition holds at $(X, Y, y)$ if and only if the condition holds at $(X, Z, y)$.

* If $X$ and $Y$ are two disjoint $C^{k}$-smooth semialgebraic sets of $\mathbf{R}^{n}$ (resp., $C^{k}$ smooth constructible sets of $\mathbf{C}^{n}$ ) such that $Y \subset \bar{X}$, the set of points, $y$, of $Y$ where the condition fails at $(X, Y, y)$ is a nowhere dense semialgebraic (resp., constructible) subset of $Y$.

If $k$ is infinity, the condition is called a stratifying condition on $\mathbf{R}$ (resp., on $\mathbf{C}$ ).

Remarks. (i) $C^{k}$-stratifications are defined in order to avoid the effective determination of the singular locus of a semialgebraic set. It leads to the question of the minimal regularity required by a stratifying condition. This is answered by the order of stratyfying condition. For constructible sets of $\mathbf{C}^{n}, C^{1}$ and $C^{\infty}$ are equivalent. Thus, only stratifying conditions are considered in the complex case.

(ii) In the complex case, the definition of Teissier [T, p. 384] does not require the constructibility of the locus of points, where the condition does not hold between two constructible sets. Here, this is done in order to allow algorithms to use formulas of $\mathbf{R}$ without checking that they define constructible sets of $\mathbf{C}^{n}$.

\subsection{Stratifying Conditions: Examples}

Definition 8. Let $k$ be an integer number or the infinity. The empty condition for $C^{k}$ regularity, $\left(\emptyset_{k}\right)$, is the condition that holds for all triplets $(X, Y, y)$ where $X$ and $Y$ are two disjoint semialgebraic sets of $\mathbf{R}^{n}$ (resp., constructible sets of $\mathbf{C}^{n}$ ) and $y$ is a point of $Y$ such that $Y \subset \bar{X}, X$ is a $C^{k}$-variety, and $y$ is a $C^{k}$-smooth point of $Y$.

Remark. The empty condition $\left(\emptyset_{k}\right)$ is a stratifying condition of order $k$ on $\mathbf{R}$.

Definition 9. Let $X$ and $Y$ be two $C^{1}$-smooth disjoint semialgebraic sets of $\mathbf{R}^{n}$ (resp., constructible sets of $\mathbf{C}^{n}$ ) such that $Y \subset \bar{X}$. Let $y$ be a point of $Y$.

The Whitney condition $(a)$ holds at $(X, Y, y)$ if and only if:

for each sequence $\left(x_{i}\right)_{i \in \mathbf{N}}$ of points of $X$ which converges to $y$ such that the sequence of tangent spaces $T_{x_{i}}^{X}$ has a limit $\tau$ in a Grassmannian $\mathbf{G}_{n, k}(\mathbf{R})\left(\operatorname{resp} ., \mathbf{G}_{n, k}(\mathbf{C})\right)$, the vector space $T_{y}^{Y}$ is a subspace of $\tau$.

The Whitney condition $(b)$ holds at $(X, Y, y)$ if and only if:

for each sequence $\left(x_{i}\right)_{i \in \mathbf{N}}$ of points of $X$ which converges to $y$ such that the sequence 
of tangent spaces $T_{x_{i}}^{X}$ has a limit $\tau$ in a Grassmannian $\mathbf{G}_{n, k}(\mathbf{R})\left(\operatorname{resp} ., \mathbf{G}_{n, k}(\mathbf{C})\right)$, and for each sequence $\left(y_{i}\right)_{i \in \mathbf{N}}$ of points of $Y$ which converges to $y$ such that the sequence of vector lines $\widehat{x_{i} y_{i}}$ has a limit $\delta$ in the projective space $\mathbf{P}_{n-1}(\mathbf{R})\left(\right.$ resp., $\left.\mathbf{P}_{n-1}(\mathbf{C})\right), \delta$ is a subspace of $\tau$.

Remark. Condition (b) implies condition (a).

The classical definition uses sequences. But, sequences are not adapted to algorithms. Then, the following equivalent definition without sequences is more convenient [BCR, p. 207].

Let $\mathbf{K}$ be $\mathbf{R}$ or $\mathbf{C}$. Let

$$
F_{a}(X)=\left\{(x, T) \in \mathbf{K}^{n} \times\left(\bigcup_{k=0}^{n} \mathbf{G}_{n, k}(\mathbf{K})\right): x \in X \text { and } T=T_{x}^{X}\right\}
$$

and

$$
F_{b}(X, Y)=\left\{(x, T, y, d) \in F_{a}(X) \times \mathbf{K}^{n} \times \mathbf{K}^{n}: y \in Y \text { and } d \in \widehat{x y}\right\} .
$$

The Whitney condition (a) holds at $(X, Y, y)$ if and only if

$$
\text { for all } \tau \text { in } \bigcup_{k=0}^{n} \mathbf{G}_{n, k}(\mathbf{K}), \quad(y, \tau) \in \overline{F_{a}(X)} \quad \Longrightarrow \quad T_{y}^{Y} \subset \tau .
$$

The Whitney condition (b) holds at $(X, Y, y)$ if and only if for all $\tau$ in $\bigcup_{k=0}^{n} \mathbf{G}_{n, k}(\mathbf{K})$ and for all $\delta$ in $\mathbf{K}^{n}, \quad(y, \tau, y, \delta) \in \overline{F_{b}(X, Y)} \quad \Longrightarrow \quad \delta \in \tau$.

The following result is required in order to show that the Whitney conditions (a) and (b) are stratifying conditions (see [T, p. 477] for the complex case).

Theorem 1. Let $X$ and $Y$ two disjoint $C^{1}$-smooth semialgebraic sets of $\mathbf{R}^{n}$ (resp., constructible sets of $\mathbf{C}^{n}$ ) such that $Y \subset \bar{X}$. Then:

(i) the set $W_{a}(X, Y)$ of points of $Y$ where the Whitney condition (a) fails at $(X, Y, y)$ is nowhere dense in $Y$; and

(ii) the set $W(X, Y)$ of points of $Y$ where the Whitney condition (b) fails at $(X, Y, y)$ is nowhere dense in $Y$.

Remarks. (i) In the complex case, the set $W(X, Y)$ is always a closed subset of $Y$, this is not always true in the real case. It has been proved by Bekka [B2], who found an example with algebraic sets of $\mathbf{R}^{n}$.

(ii) The Whitney conditions are stratifying conditions of order 1 on $\mathbf{R}$ and on $\mathbf{C}$.

All semialgebraic maps can be stratified. Sometimes this description is not sufficient. For certain maps, it is possible to require more. 
Definition 10 [Th, p. 57], see also [GWdPL, p. 23]. Let $f:(V, \mathcal{S}) \longrightarrow\left(W, \mathcal{S}^{\prime}\right)$ be a $C^{1}$-stratified map, let $(X, Y)$ be a pair of strata of $\mathcal{S}$, and let $y$ be a point of $\bar{X} \cap Y$.

Condition $\left(a_{f}\right)$ holds at $(X, Y, y)$ if and only if:

for all sequences, $\left(x_{i}\right)_{i \in \mathbf{N}}$, of points of $X$ which converge to $y$ and such that the sequence of the kernels of the differential map of $\left.f\right|_{X}$ at the points $x_{i}$, $\left(\operatorname{Ker} \partial_{x_{i}}\left(\left.f\right|_{X}\right)\right)_{i \in \mathbf{N}}$, converges in some Grassmannian to $\tau$, Ker $\partial_{y}\left(\left.f\right|_{Y}\right)$ is a subspace of $\tau$.

Condition $\left(a_{f}\right)$ holds at the pair $(X, Y)$ if and only if it holds at $(X, Y, y)$ for all points $y$ in $\bar{X} \cap Y$.

The map $f:(V, \mathcal{S}) \rightarrow\left(W, \mathcal{S}^{\prime}\right)$ is a Thom's map if and only if condition $\left(\mathrm{a}_{\mathrm{f}}\right)$ holds for all pairs of strata of $\mathcal{S}$.

Remark. In order to use condition ( $\mathrm{a}_{\mathrm{f}}$ ) algorithmically, an equivalent condition without sequences is more convenient. Let

$$
F(X)=\left\{(x, T) \in \mathbf{R}^{n} \times\left(\bigcup_{k=0}^{n} \mathbf{G}_{n, k}(\mathbf{R})\right): x \in X \text { and } T=\operatorname{Ker} \partial_{x}\left(\left.f\right|_{X}\right)\right\} .
$$

condition $\left(\mathrm{a}_{\mathrm{f}}\right)$ holds at $(X, Y, y)$ if and only if

$$
\text { for all } \tau \text { of } \bigcup_{k=0}^{n} \mathbf{G}_{n, k}(\mathbf{R}), \quad(y, \tau) \in \overline{F(X)} \quad \Longrightarrow \quad \operatorname{Ker} \partial_{y}\left(\left.f\right|_{Y}\right) \subset \tau \text {. }
$$

Definition 11 [B1, p. 13]. Let $X$ and $Y$ two disjoint $C^{1}$-smooth semialgebraic subsets of a semialgebraic set $V$ of $\mathbf{R}^{n}$ such that $Y \subset \bar{X}$. Let $y$ be a point of $Y$. Let $U$ be a neighborhood of $y$ in $V$ and let $f_{Y}$ be a $C^{1}$-smooth map from $U$ to $\mathbf{R}^{+}$such that $f_{Y}^{-1}(0)=Y$.

Condition (C) for the map $f_{Y}$ holds at $(X, Y, y)$ if and only if there exists a neighborhood $U^{\prime}$ of $y$ in $V$ such that

$$
\left.f_{Y}\right|_{(X \cup Y) \cap U^{\prime}}:\left(X \cap U^{\prime}, Y \cap U^{\prime}\right) \longrightarrow\left(\mathbf{R}^{+} \backslash\{0\},\{0\}\right)
$$

is a $C^{1}$-stratified map for which condition $\left(\mathrm{a}_{\mathrm{f}}\right)$ holds at $y$.

Condition (C) is weaker than the Whitney condition (b) in general. But, it is sufficient to imply the locally topological triviality. Trotman [Tr] has proved the following result which is an excellent illustration of the connection between the two conditions of regularity.

Theorem 2. Let $\mathcal{S}$ be a stratification of a semialgebraic set $V$ of $\mathbf{R}^{n}$. Let $Y$ be a stratum of $\mathcal{S}$ and $y$ be a point of $Y$. Let $\operatorname{St}(Y)$ be the star of $Y$ (the set of all the strata of $\mathcal{S}$ with Euclidean closure containing $Y$ ). The two following conditions are equivalent:

(i) For each stratum $X$ of $\operatorname{St}(Y)$, the Whitney conditions hold at $(X, Y, y)$.

(ii) For all tubular functions $f_{Y}$ (i.e., $f_{Y}=\sum_{i=\operatorname{dim}_{y} Y+1}^{\operatorname{dim} V} x_{i}^{2}$ in a local map of $Y$ in $V$ ), condition $(C)$ for $f_{Y}$ holds at $(X, Y, y)$. 


\subsection{Canonical Stratification}

Definition 12 [M, p. 210]. Let $k$ be an integer number or infinity. Let $\mathcal{S}$ and $\mathcal{S}^{\prime}$ be two $C^{k}$-stratifications of a semialgebraic set of $\mathbf{R}^{n}$ (resp., a constructible set of $\mathbf{C}^{n}$ ). For each integer number $m$ between 0 and $n$, let $S^{m}$ be the set of all the strata of $\mathcal{S}$ of dimension $m$ and let $S^{\prime m}$ be the set of all the strata of $\mathcal{S}^{\prime}$ of dimension $m$.

$\mathcal{S}<\mathcal{S}^{\prime}$ if and only if there exists an integer number $d$ such that:

(i) $S^{m}=S^{\prime m}$ for all integer numbers $m$ between $d+1$ and $n$; and

(ii) $S^{d} \supset S^{\prime d}$ and $S^{d} \neq S^{\prime d}$.

Definition 13. Let $V$ be a semialgebraic set of $\mathbf{R}^{n}$ (resp., a constructible set of $\mathbf{C}^{n}$ ). Let $k$ be an integer number or the infinity. Let $(I)$ be a stratifying condition of order $k^{\prime}$ on $\mathbf{R}$ (resp., on $\mathbf{C}$ ) where $k^{\prime} \leq k$. A canonical (I)-regular $C^{k}$-stratification of a set $V$ is a minimal (I)-regular $C^{k}$-stratification of the set $V$.

All stratifications constructed in this paper are canonical. The following result shows how canonical stratifications can be computed.

Theorem 3. Let $V$ be a semialgebraic set of $\mathbf{R}^{n}$ (resp., a constructible set of $\mathbf{C}^{n}$ ). Let (I) be a stratifying condition on $\mathbf{R}$ (resp., on $\mathbf{C}$ ) of order $k^{\prime}$. Let $k$ be infinity or an integer number greater than or equal to $k^{\prime}$. Then:

(i) There exists a unique canonical (I)-regular $C^{k}$-stratification of the set $V$.

(ii) The canonical (I)-regular $C^{k}$-stratification of the set $V$ can be constructed by means of a filtration by closed subsets of $V$

$$
F_{0}=V \supset F_{1} \supset \cdots \supset F_{i} \supset \cdots \supset F_{l}=\emptyset
$$

defined by

$$
\left\{\begin{aligned}
& F_{1}=F_{0, \operatorname{sing} k}= V_{\text {sing } k} \text { and for } \quad i=2, \ldots, l, \\
& F_{i}=F_{i-1, \text { sing } k} \cup\left[\overline{\bigcup_{C \in \mathcal{S}_{i-1}} I\left(C,\left(F_{i-1, \text { reg } k} \cap \bar{C}\right)_{\operatorname{reg} k}\right)} \cap V\right] \\
& \cup\left[\bigcup_{C \in \mathcal{S}_{i-1}}\left(\bar{C} \cap \overline{\left(F_{i-1} \backslash \bar{C}\right)} \cap V\right)\right],
\end{aligned}\right.
$$

where:

* $\mathcal{S}_{i}$ is the set of all the connected components of the $F_{j} \backslash F_{j+1}$ for $j$ from 0 to $i-1$. This is the canonical (I)-regular $C^{k}$-stratification of $V \backslash F_{i}$.

$* I(A, B)$ represents the set of points $y$ of $B$ where Condition (I) fails at $(A, B, y)$.

The canonical (I)-regular $C^{k}$-stratification of the set $V$ is the partition $\mathcal{S}_{l}$. The filtration $F_{0}=V \supset F_{1} \supset \cdots \supset F_{i} \supset \cdots \supset F_{l}=\emptyset$ is called the filtration associated to the canonical (I)-regular $C^{k}$-stratification of the set $V$. 
Remarks. (i) [GWdPL, p. 20] gives a proof of this theorem when Condition $(I)$ is the Whitney condition (b), $V$ is a semialgebraic set of $\mathbf{R}^{n}, k$ is infinity. The chosen definition of stratifications allows partitions without boundary condition and not connected strata.

(ii) In order to get a canonical $(I)$-regular $C^{k}$-stratification of the set $V$, at each step of the construction of the filtration, only the points, where problems in the stratification process occur, are removed. These "bad" points constitute the next element of the associated filtration. This set is rare because Condition $(I)$ is a stratifying condition on $\mathbf{R}$ (resp., on $\mathbf{C}$ ) of order $k$. This set can be constructed by:

(a) The points are not $C^{k}$. They constitute the set $F_{i-1, \operatorname{sing} k}$.

(b) The points where the boundary condition fails. They constitute the set

$$
\bigcup_{C \in \mathcal{S}_{i-1}}\left(\bar{C} \cap \overline{\left(F_{i-1} \backslash \bar{C}\right)} \cap V\right) .
$$

(c) The points where Condition (I) fails. They constitute the set

$$
\bigcup_{C \in \mathcal{S}_{i-1}} I\left(C,\left(F_{i-1, \operatorname{reg} k} \cap \bar{C}\right)_{\operatorname{reg} k}\right) .
$$

This set is not always closed in $V$. In order to get strata which are $C^{k}$-varieties, it is necessary to take its Euclidean closure in $V$.

Proof. Let $\mathcal{S}=\mathcal{S}_{l}$. Teissier [T, pp. 384-386] showed in the complex case and for $k$ infinity that the partition $\mathcal{S}_{l}$ is a $(I)$-regular stratification of the set $V$. The same arguments lead to the general case of this theorem.

* Canonicity of the Stratification $\mathcal{S}_{l}$

Let $\mathcal{S}^{\prime}$ be an $(I)$-regular $C^{k}$-stratification of the set $V$. Let $d$ be an integer number between 0 and $n$.

Hypotheses: The strata of $\mathcal{S}$ and of $\mathcal{S}^{\prime}$, having dimensions strictly greater than $d$, coincide. Let $Y$ be a stratum of $\mathcal{S}^{\prime}$ of dimension $d$. To prove that $\mathcal{S}$ is the canonical (I)-regular $C^{k}$-stratification of $V$, is to prove with the last hypotheses that $Y$ is contained in a stratum of $\mathcal{S}$. Let $i$ be the integer number such that $Y \subset F_{i-1}$ and $Y \not \subset F_{i}$.

First Step: $Y$ is an open set of $F_{i-1}$.

Let $y$ be a point of $Y$. If $Y$ is not a neighborhood of $y$ in $F_{i-1}$, then:

The partition $\mathcal{S}^{\prime}$ is finite, hence there is a stratum $W$ of $\mathcal{S}^{\prime}$ different from $Y$ and a sequence of points of $F_{i-1} \cap W$ which converges to $y$. But $y \in \bar{W} \cap Y$. The boundary condition between strata of $\mathcal{S}^{\prime}$ implies that $Y \subset \bar{W} \backslash W$ and $\operatorname{dim} W>d$.

Then, $W$ is a stratum of $\mathcal{S}$ as well. This is a connected component of a $F_{i^{\prime}-1} \backslash F_{i^{\prime}}$ for some $i^{\prime}$. But $W \cap F_{i-1} \neq \emptyset$. Therefore, by the construction of $\mathcal{S}, i^{\prime}$ is larger than or equal to $i$.

But, $Y \subset \bar{W} \backslash W \subset F_{i^{\prime}} \subset F_{i}$. This is impossible. Thus, $Y$ is open in $F_{i-1}$.

Second Step: $Y$ is a subset of $F_{i-1} \backslash F_{i}$.

(i) Let $X$ be a connected component of $F_{j-1} \backslash F_{j}$ with $j<i-1$ such that $y \in \bar{X}$. The partition $\mathcal{S}^{\prime}$ is finite. Hence, there is a stratum $W$ of $\mathcal{S}^{\prime}$ and a sequence of points $X \cap W$ 
converging to $y$. But $y \in \bar{W}$. The boundary condition between the strata of $\mathcal{S}^{\prime}$ implies that $Y \subset \bar{W} . X \cap Y=\emptyset$ where $Y \subset F_{i} \subset F_{j}$. Then $Y \neq W$. Therefore, $Y \subset \bar{W} \backslash W$ and $\operatorname{dim} W>d$. The set $W$ is also a stratum of $\mathcal{S}$, and it proves that $X=W$. Then, $X$ is a stratum of $\mathcal{S}^{\prime}$.

Let $x \in F_{i} \cap Y$.

(ii) If $x \in F_{i-1, \operatorname{sing} k}$ the set $Y$ is an open set of $F_{i-1}, y \in Y_{\operatorname{sing} k}$. This is impossible because $Y$ is a $C^{k}$-manifold.

(iii) If $x \in \bar{C} \cap\left(\overline{F_{i-1} \backslash \bar{C}}\right)$ for some connected component, $C$, of some $F_{j-1} \backslash F_{j}$ where $j<i-1: Y$ is an open set of $F_{i-1}$ which contains $x$. Hence, $x \in \bar{C} \cap(\overline{Y \backslash \bar{C}})$. The argument (i) applied to $C$ shows that $C$ is a stratum of $\mathcal{S}^{\prime}$. This is impossible because of the boundary condition of $\mathcal{S}^{\prime}$.

(iv) If $x \in I\left(C,\left(F_{i-1, \operatorname{reg} k} \cap \bar{C}\right)_{\operatorname{reg} k}\right)$ for some connected component, $C$, of some $F_{j-1} \backslash F_{j}$ where $j<i-1$. If $x$ is not $C^{k}$-smooth in $F_{i-1, \operatorname{reg} k} \cap \bar{C}$, then $x$ is not $C^{k}$ smooth in $F_{i-1}$; or $x$ is a point such that $\bar{C}$ is not a neighborhood of $x$ in $F_{i-1}$, i.e., a point of $\bar{C} \cap\left(\overline{F_{i-1} \backslash \bar{C}}\right)$.

The arguments (i) and (ii) show that in this last case $x \notin Y$.

Then, $\bar{C}$ is a neighborhood of $x$ in $\left(F_{i-1, \operatorname{reg} k} \cap \bar{C}\right)_{\operatorname{reg} k}$ and thus, Condition $(I)$ fails at $\left(C, F_{i-1, \operatorname{reg} k} \cap \bar{C}, x\right)$. Condition $(I)$ is a stratifying condition on $\mathbf{R}$ (resp., $\mathbf{C}$ ). The set $Y$ is an open subset of $F_{i-1}$ which contains $x$. Then, Condition $(I)$ fails at $(C, Y \cap \bar{C}, x)$. Argument (i) applied to $C$ shows that $C$ is a stratum of $\mathcal{S}^{\prime}$. The boundary condition between the strata of $\mathcal{S}^{\prime}$ implies that $Y \subset \bar{C}$. Then $x \in I(C, Y)$. This is impossible because of the $(I)$-regularity of $\mathcal{S}^{\prime}$.

(v) Let:

$$
\begin{aligned}
G_{i}=F_{i-1, \operatorname{sing} k} \cup & {\left[\bigcup_{C \in \mathcal{S}_{i-1}} I\left(C,\left(F_{i-1, \mathrm{reg} k} \cap \bar{C}\right)_{\mathrm{reg} k}\right) \cap V\right] } \\
\cup & {\left[\bigcup_{C \in \mathcal{S}_{i-1}}\left(\bar{C} \cap \overline{\left(F_{i-1} \backslash \bar{C}\right)} \cap V\right)\right] . }
\end{aligned}
$$

Then $Y \cap G_{i}=\emptyset . Y$ is an open subset of $F_{i-1}, Y \cap \overline{G_{i}}=\emptyset$. Then $Y \cap F_{i}=\emptyset$. The connectedness of $Y$ implies that $Y$ is contained in some connected component of $F_{i-1} \backslash F_{i}$ of dimension $d$.

Conclusion. Each stratum of dimension $d$ of $\mathcal{S}^{\prime}$ is contained in some stratum of dimension $d$ of $\mathcal{S}$. The partition $\mathcal{S}$ of $V$ is the canonical $(I)$-regular $C^{k}$-stratification of $V$.

Remarks. (i) The filtration $F_{0}=V \supset F_{1} \supset \cdots \supset F_{i} \supset \cdots \supset F_{l}=\emptyset$ is called the associated filtration to the canonical (I)-regular $C^{k}$-stratification of set $V$. The number of nonempty sets of the associated filtration $l$ is called the depth of the associated filtration to the canonical ( $I$ )-regular $C^{k}$-stratification of set $V$.

(ii) For each $i$ from 1 to $l, F_{i}$ is a nowhere-dense subset of $F_{i-1}$, thus $\operatorname{dim} F_{i}<$ $\operatorname{dim} F_{i-1}$. Then, $l-1 \leq \operatorname{dim} V$. There is at most $\operatorname{dim} V+1$ nonempty elements in the associated filtration to the canonical (I)-regular $C^{k}$-stratification of set $V$. 


\section{Complexity and Basic Constructions}

\subsection{The Model of Complexity}

Definition 14. The degree of a formula of $\mathbf{R}$ is the maximum of 2 and of the sum of all total degrees of polynomials from the formula.

All algorithms in this paper have as inputs quantifier-free formulas of $\mathbf{R}$. In order to estimate algorithm complexities, parameters in input must be chosen. The complexity model used here is an easy one. Only two parameters are considered. The first, $D$, is an integer number greater than the maximum of 2 and the sum of all degrees of the input formulas. The integer number $D$ is not a parameter of sets but just a parameter of quantifier-free formulas of $\mathbf{R}$. Every semialgebraic set of $\mathbf{R}$ can be defined by formulas with various degrees. The second parameter, $n$, is just the dimension of the surrounding affine space $\mathbf{R}^{n}$ of the input set. This is the number of variables in the input formulas. The pair $(n, D)$ is called the input size.

Each input and output polynomial is represented by the sequence of all its coefficients (dense representation).

In order to compute complexities, all algorithms are considered as arithmetic networks.

Definition 15. Let $\mathbf{A}$ be an ordered ring. An arithmetic network on $\mathbf{A}$ is an oriented acyclic graph where:

(i) each vertex represents a processor which makes:

an arithmetic operation in the ring $\mathbf{A}$, or

a comparison between two elements of $\mathbf{A}$, or

a Boolean operation, or

a selection according to the value of a Boolean variable, or

an introduction of a constant from $\mathbf{A}$; and

(ii) each edge notes the sending of an output from a processor to another processor.

Definition 16 [vzG]. The sequential complexity of an arithmetic network is the total number of edges of the network considered as a graph. The parallel complexity of an arithmetic network is the depth of the network viewed as an acyclic graph.

The sequential complexity of an algorithm is a function $S C$ of the input size defined by:

$S C(n, D)$ is the maximum of all sequential complexities of the arithmetic networks representing the algorithm for input of size $(n, D)$.

The parallel complexity of an algorithm is a function $P C$ of the input size defined by:

$P C(n, D)$ is the maximum of all parallel complexities of the arithmetic networks representing the algorithm for input of size $(n, D)$.

Definition 17. An algorithm is well parallelizable if and only if its parallel complexity is at most $(\log (C))^{O(1)}$ where $C$ is its sequential complexity. 
Remark. All algorithms built in this paper are well parallelizable. They use only constructions that lead to linear algebra. Thus, all computations are done in the ring of coefficients of the input polynomials. All algorithms, like the polynomial factorization, which are not well parallelizable or which need operations not in the ring of coefficients, are not used here. For example, the result of Chistov and Grigoryev [CG] about the determination of irreducible components of a projective algebraic set uses a factorization algorithm, for which no well-parallelizable versions are known. In all proofs, only sequential complexities are studied in detail. Parallel complexities are obtained mechanically.

Definition 18. An algorithm is admissible if and only if it is well parallelizable and, if for all inputs of size $(n, D)$, it can be represented by an arithmetic network of sequential complexity at most $D^{n^{O(1)}}$.

\subsection{Known Algorithmic Results}

The aim of this section is to present all algorithmic results used in this paper in order to build stratification algorithms for semialgebraic sets of $\mathbf{R}^{n}$ and for constructible sets of $\mathbf{C}^{n}$. The main algorithmic tool used is the fast quantifier elimination algorithm (Theorem 4). It is an efficient version of the Tarski-Seidenberg principle. Using this result a lot of admissible algorithms can be constructed.

Definition 19. A formula of $\mathbf{R}$ is a prenex formula if and only if it can be written $b_{1} b_{2} \cdots b_{r} \Psi$ such that:

(i) for all $i$ from 1 to $r, b_{i}=\forall v_{1}^{i} \cdots \forall v_{j}^{i} \cdots \forall v_{n_{i}}^{i}$ or $b_{i}=\exists v_{1}^{i} \cdots \exists v_{j}^{i} \cdots \exists v_{n_{i}}^{i}$, where all $v_{j}^{i}$ for $j=1, \ldots, n_{i}$ are variables; and

(ii) $\Psi$ does not contain any quantifiers.

The number, $s$, of quantifier blocks of a prenex formula of $\mathbf{R}$ is the smallest integer $r$ such that (i) and (ii) hold. For $i$ from 1 to $s$ the integer number $n_{i}$ is the size of the $i$ th quantifier block.

Theorem 4 [R], see also [HRS1]. Let $\Phi$ be a prenex formula of $\mathbf{R}$ with coefficients in a subring $\mathbf{A}$ of $\mathbf{R}$. Let $r$ be the number of quantifier blocks of $\Phi$ and let $n_{1}, \ldots, n_{r}$ be the sizes of the quantifier blocks. Let $n_{0}$ be the number of free variables of $\Phi$ (i.e., the number of variables which are not in the quantifier blocks).

Then, there exists a well-parallelizable arithmetic network on $\mathbf{A}$ with a sequential complexity $D^{\Pi_{i=0}^{r} O\left(n_{i}\right)}$ which constructs a quantifier-free formula of $\mathbf{R}$ equivalent to $\Phi$ (i.e., both formulas define the same semialgebraic set of $\mathbf{R}^{n_{0}}$ ).

Remark. In the general case the real quantifier elimination is doubly exponential in the number of variables. It has been proved by an example found by Davenport and Heintz $[\mathrm{DH}]$. However, in a lot of useful situations, the number of quantifier blocks is fixed and is 
independent of the number of variables. In these cases the fast real quantifier elimination leads to admissible algorithms (singly exponential in the number of variables).

The main tool used to prove the latter theorem is the following empty set test.

Theorem 5 [R], see also [GV]. Let $V$ be a semialgebraic set of $\mathbf{R}^{n}$ defined by a quantifier-free formula of $\mathbf{R}$ with coefficients in a subring $\mathbf{A}$ of $\mathbf{R}$ and of degree at most $D$. Then, there exists a well-parallelizable arithmetic network on $\mathbf{A}$ with a sequential complexity $D^{O(n)}$ which determines whether $V=\emptyset$ or not.

Strata of canonical stratification are defined as connected components. Thus, the next result is an important one.

Theorem 6 [CGV], [HRS2], see also [GHRSV]. Let $V \subset \mathbf{R}^{n}$ be a semialgebraic set defined by a quantifier-free formula of $\mathbf{R}$ of degree at most $D$ and with coefficients in $a$ subring $\mathbf{A}$ of $\mathbf{R}$. Then, there exists a well-parallelizable arithmetic network on $\mathbf{A}$ with a sequential complexity $D^{n^{o(1)}}$ which constructs quantifier-free formulas of $\mathbf{R}$ which define every connected component of $V$.

\subsection{Other Algorithmic Tools}

Translations of elementary geometrical constructions into formulas of $\mathbf{R}$ are presented in this subsection. They are used throughout this paper.

Lemma 1. Let $V$ be a semialgebraic set of $\mathbf{R}^{n}$ defined by a quantifier-free formula of $\mathbf{R}$ of degree at most $D$. Let $x$ be a point of $V$. Then the dimension of $V$ at the point $x$, $\operatorname{dim}_{x} V$, is characterized by

$$
\begin{aligned}
\operatorname{dim}_{x} V \geq d \Longleftrightarrow & \forall \eta>0, \\
& \exists x^{\prime} \in B(x, \eta) \cap V, \forall \varepsilon>0, \exists \pi \in \mathbf{M}_{n, d}(\mathbf{R}), \\
& \forall z \in B\left(\pi\left(x^{\prime}\right), 1\right), \exists y \in B\left(x^{\prime}, \varepsilon\right) \cap V, \quad \pi(y)=z,
\end{aligned}
$$

where $\mathbf{M}_{n, d}(\mathbf{R})$ denotes the set of real matrices with d lines and $n$ rows.

This is a formula of $\mathbf{R}$ equivalent to a prenex formula of $\mathbf{R}$ with six quantifier blocks, respectively, of size $1, n, 1, n d, d, n$ and of degree at most $2 D+d+8$.

Proof. The set $V$ is the finite union of semialgebraic sets $V_{i}$ semialgebraically homeomorphic to $] 0,1\left[^{d_{i}}\right.$ for some integer number $d_{i}$ (where $] 0,1\left[^{0}\right.$ denotes $\{0\}$ here).

The dimension at $x$ of $V$ is larger than or equal to $d$ if and only if $x$ is in the Euclidean closure of some $V_{i}$ for some $d_{i} \geq d$. Thus, for every neighborhood of $x$, there is a point $x^{\prime}$ of $V$ such that for every neighborhood $U$ of $x^{\prime}$, there is a linear map $\pi: \mathbf{R}^{n} \mapsto \mathbf{R}^{d}$ such that $\left.\pi\right|_{U}$ maps $U$ surjectively on the unit ball centered at $\pi\left(x^{\prime}\right)$.

The expected formula is just the translation of the described property. 
Lemma 2. Let $V$ be a semialgebraic set of $\mathbf{R}^{n}$ defined by a quantifier-free formula of $\mathbf{R}$ of degree at most $D$. Let $x$ be a point of $V$. Then, the secants limits set of $V$ at $x$, called $\operatorname{limsec}_{x}(V)$, is defined by the following formula of $\mathbf{R}$ :

$$
\begin{aligned}
t \in \operatorname{limsec}_{x}(V) \Longleftrightarrow \forall \eta> & 0, \forall \varepsilon>0, \\
& \exists u, v \in B(x, \eta) \cap V, \exists \lambda \in \mathbf{R},\|t-\lambda(u-v)\|^{2} \leq \varepsilon,
\end{aligned}
$$

where $B(x, \eta)$ is the ball centered at $x$ and of radius $\eta$.

This is a formula of $\mathbf{R}$ with two quantifier blocks, respectively, of size $2,2 n+1$, and with $n$ free variables. Its total degree is at most $2 D+8$.

Proof. Due to the definition of secants limits

$$
\operatorname{limsec}_{x}(V)=\bigcap_{\eta>0} \overline{\left\{\lambda(u-v) \in \mathbf{R}^{n}: \lambda \in \mathbf{R} u, v \in V \cap B(x, \eta)\right\}} .
$$

The translation of the intersection and of the Euclidean closure leads to the formula of the lemma.

Remarks. (i) If $x$ is $C^{1}$-smooth point of $V$, the set $\operatorname{limsec}_{x}(V)$ is just the tangent space $T_{x}^{V}$ of $V$ at $x$.

(ii) The set, $\operatorname{limsec}_{x}(V)$ is not always a vector space. See, for example,

$$
V=\left\{\left(x_{1}, x_{2}\right): x_{1} \geq 0, x_{2} \geq 0\right\} \quad \text { and } \quad x=(0,0) .
$$

Lemma 3. Let $f: D_{f} \rightarrow \mathbf{R}^{p}$ be a semialgebraic map defined on an open semialgebraic set $D_{f} \in \mathbf{R}^{n}$ by its graph, graph $(f)$ as a semialgebraic set of $\mathbf{R}^{n+p}$ by a quantifier-free formula of $\mathbf{R}$ of degree at most $D$.

Let $J_{f}$ be the following semialgebraic set:

$$
J_{f}=\left\{\begin{array}{l}
(x, A) \in \mathbf{R}^{n} \times \mathbf{M}_{n, p}(\mathbf{R}): \exists \varepsilon>0, \exists C>0, \forall x^{\prime} \in B(x, \varepsilon), \\
\forall f(x) \in \operatorname{graph}(f) \cap\left(\{x\} \times \mathbf{R}^{p}\right), \forall f\left(x^{\prime}\right) \in \operatorname{graph}(f) \cap\left(\left\{x^{\prime}\right\} \times \mathbf{R}^{p}\right) \\
\left.\sum_{i=1}^{p}\left\|f_{i}(x)-f_{i}\left(x^{\prime}\right)-A_{i} \cdot{ }^{t}\left(x-x^{\prime}\right)\right\|^{2}<C\left\|x-x^{\prime}\right\|^{4}\right\},
\end{array}\right.
$$

where $f_{i}(x)$ is the ith coordinate of $f(x)$ in $\mathbf{R}^{p},{ }^{t}\left(x-x^{\prime}\right)$ is the column vector of $\mathbf{R}^{p}$ containing the $x_{i}-x_{i}^{\prime}, B(x, \varepsilon)$ is the ball centered at $x$ and of radius $\varepsilon$, and $A_{i}$ represents the ith line of the matrix $A$ in $\mathbf{M}_{n, p}(\mathbf{R})$.

Then, the semialgebraic set $J_{f}$ is characterized by: for every $x \in D_{f}$ :

$$
\begin{cases}J_{f} \cap\left(\{x\} \times \mathbf{M}_{n, p}(\mathbf{R})\right)=\left\{\left(x, J_{f, x}\right)\right\} & \text { if the Jacobian matrix exists and is } J_{f, x}, \\ J_{f} \cap\left(\{x\} \times \mathbf{M}_{n, p}(\mathbf{R})\right)=\emptyset & \text { otherwise. }\end{cases}
$$

The formula defining $J_{f}$ is equivalent to a prenex formula of $\mathbf{R}$ with two quantifier blocks, respectively, of size $2, n+2 p$, and with $n+n p$ free variables. Its degree is at most $2 D+8$. 
Proof. Translating into a formula of $\mathbf{R}$ the definition of the differential map of a map at a point is enough to get the expected formula.

Many stratifying conditions use limits of vector spaces in Grassmannians. Each point of a Grassmannian corresponds to a vector space. The Grassmannian of all vector subspaces of $\mathbf{R}^{n}$ of dimension $k$ can be represented as an affine algebraic variety of $\mathbf{R}^{n^{2}}$ by

$$
\mathbf{G}_{n, k}(\mathbf{R})=\left\{A \in \mathbf{M}_{n, n}(\mathbf{R}): A={ }^{t} A, A^{2}=A, \operatorname{trace}(A)=k\right\} .
$$

In this representation, a vector subspace $T$ of $\mathbf{R}^{n}$ is associated to the matrix of $\mathbf{M}_{n, n}(\mathbf{R})$ in the canonical basis of $\mathbf{R}^{n}$ of the orthogonal projection on $T$ (see [BCR, p. 65]). However, vector spaces are often built as semialgebraic subsets of $\mathbf{R}^{n}$ by a formula of $\mathbf{R}$. Thus, a bridge between the two representations is useful.

Lemma 4. Let $T$ be a vector subspace of $\mathbf{R}^{n}$ of dimension $k$ defined by a quantifier-free formula of $\mathbf{R}$ of degree at most $D$. Then the point $A=\left(a_{i, j}\right)_{i, j}$ of $\mathbf{G}_{n, k}(\mathbf{R})$ corresponding to the vector space $T$ is defined by the following formula of $\mathbf{R}$ :

$\forall\left(t_{1}, \ldots, t_{n}\right) \in T \quad\left(\sum_{i=1}^{n} a_{i, j} t_{i}=t_{j}\right.$ and $\left(a_{1, j}, \ldots, a_{n, j}\right) \in T$ for all $\left.j=1, \ldots, n\right)$.

This is a formula of $\mathbf{R}$ with a single quantifier block of size $n$, with $n^{2}$ free variables and of degree at most $(n+1) D+2 n$.

Proof. The formula is just the translation of the correspondence of a vector subspace and the matrix of the projection on this subspace.

\section{4. $C^{1}$-Locus}

The aim of this section is to get a quantifier-free formula of $\mathbf{R}$ defining the set of points of a semialgebraic set which are not $C^{1}$-smooth (Proposition 2). This result and the canonical stratification algorithm (Theorem 8) lead to an algorithm for the computation of the canonical $C^{1}$-stratification of a semialgebraic set of $\mathbf{R}^{n}$.

Proposition 2. Let $V$ be a semialgebraic subset of $\mathbf{R}^{n}$ defined by a quantifier-free formula of $\mathbf{R}$ of degree at most $D(D \geq n)$ and with coefficients in a subring $\mathbf{A}$ of $\mathbf{R}$. Then, there exists a well-parallelizable arithmetic network on $\mathbf{A}$ with a sequential complexity $D^{n^{o(1)}}$ which constructs a quantifier-free formula of $\mathbf{R}$ defining the semialgebraic set, $V_{\text {sing } 1}$, of points of $V$ which are not $C^{1}$-smooth in $V$.

The proof provides the expected algorithm.

Proof. Lemma 2 provides a formula of $\mathbf{R}$ which defines the secants limits $\operatorname{set} \operatorname{limsec}_{x}(V)$, at a point $x$ of $V$. The fast quantifier elimination algorithm (Theorem 4) gives a quantifierfree formula of $\mathbf{R}$, with a sequential complexity $D^{O\left(n^{2}\right)}$, which defines $\operatorname{limsec}_{x}(V)$ where $x$ is seen as a parameter. 
If this set $\operatorname{limsec}_{x}(V)$ is not a vector space, $x$ is not $C^{1}$-smooth in $V$. These points are characterized by

$$
\exists t, t^{\prime} \in \operatorname{limsec}_{x}(V), \quad t+t^{\prime} \notin \operatorname{limsec}_{x}(V) .
$$

This is a prenex formula of $\mathbf{R}$ with $n$ free variables, with a single quantifier block of size $2 n$ and of degree at most $3 D^{O\left(n^{2}\right)}$. A quantifier-free formula of $\mathbf{R}$ is constructed, which defines those points, with a sequential complexity $\left[3 D^{O\left(n^{2}\right)}\right]^{O\left(n^{2}\right)}=D^{O\left(n^{4}\right)}$ by means of the fast quantifier elimination algorithm (Theorem 4).

For each other point $x, \operatorname{limsec}_{x}(V)$ is equal to the tangent space $T_{x}^{V}$, of $V$ at $x$. Let $x$ be such a point. The point $x$ of $V$ is $C^{1}$-smooth in $V$ if and only if the orthogonal projection $\pi_{x}$ from $V$ to the affine space $\left\{x+t \in \mathbf{R}^{n}: t \in T_{x}^{V}\right\}$ is locally bijective at $x$. Only the surjectivity is necessary. Indeed, Let $x$ be a point such that the orthogonal projection $\pi_{x}$ from $V$ to $\left\{x+t \in \mathbf{R}^{n}: t \in T_{x}^{V}\right\}$ is locally surjective, but not injective at $x$. Then, there are two sequences of point of $V,\left(u_{i}\right)_{i \in \mathbf{N}}$ and $\left(v_{i}\right)_{i \in \mathbf{N}}$, each of them converging to $x$ and such that $\pi_{x}\left(u_{i}\right)=\pi_{x}\left(v_{i}\right)$ and $u_{i} \neq v_{i}$ for all integer numbers $i$. For all integer numbers $i, u_{i}-v_{i} \in \operatorname{Ker} \pi_{x}=\left(T_{x}^{V}\right)^{\perp}$. But, by the compactness of $\mathbf{P}_{n-1}(\mathbf{R})$, there is a subsequence of $\left(\widehat{u_{i} v_{i}}\right)_{i \in \mathbf{N}}$ which converges to a line $l$. But, $l \subset T_{x}^{V} \cap\left(T_{x}^{V}\right)^{\perp}=\{0\}$ which is impossible.

Thus, $C^{1}$-smooth points $x$ are characterized by

$$
\begin{array}{r}
\forall \varepsilon>0, \exists \eta>0,\left.\quad \pi_{x}\right|_{B(x, \varepsilon) \cap V}: B(x, \varepsilon) \cap V \rightarrow\left\{x+t \in \mathbf{R}^{n}: t \in T_{x}^{V}\right\} \\
\text { is surjective on } \quad B(x, \eta) \cap\left\{x+t \in \mathbf{R}^{n}: t \in T_{x}^{V}\right\} .
\end{array}
$$

By translation into formula, this is equivalent to

$$
\forall \varepsilon>0, \exists \eta>0, \forall t^{\prime} \in T_{x}^{V}, \exists x^{\prime} \in B(x, \varepsilon) \cap V, \quad \pi_{x}\left(x^{\prime}\right)=x+t^{\prime} .
$$

The projection $\pi_{x}$ is defined by

$$
\begin{aligned}
& \pi_{x}(y)=z \quad \Longleftrightarrow \exists h \in\left(T_{x}^{V}\right)^{\perp}, \quad z=y-h \text { and } z-x \in T_{x}^{V}, \\
& \pi_{x}(y)=z \quad \Longleftrightarrow \exists h \in \mathbf{R}^{n}, \forall t \in T_{x}^{V}, \quad \sum_{i=1}^{n} h_{i} t_{i}=0 \text { and } z=y-h \text { and } z-x \in T_{x}^{V} .
\end{aligned}
$$

After translation of the projection $\pi_{x}$, the formula turns into

$$
\begin{gathered}
\forall \varepsilon>0, \exists \eta>0, \forall t^{\prime} \in T_{x}^{V}, \exists x^{\prime} \in B(x, \varepsilon) \cap V, \exists h \in \mathbf{R}^{n}, \forall t \in T_{x}^{V} \\
\sum_{i=1}^{n} h_{i} t_{i}=0 \quad \text { and } \quad x^{\prime}-h=x+t^{\prime} .
\end{gathered}
$$

This is equivalent to a prenex formula of $\mathbf{R}$ with five quantifier blocks, respectively, of size $1,1, n, 2 n, n$ and with $n$ free variables. The degree is at most $D^{O\left(n^{2}\right)}$. The fast quantifier elimination algorithm (Theorem 4 ) builds, with a sequential complexity $\left[D^{O\left(n^{2}\right)}\right]^{O\left(n^{4}\right)}=$ $D^{O\left(n^{6}\right)}$, an equivalent quantifier-free formula of $\mathbf{R}$. Therefore, this algorithm gives, with a sequential complexity $D^{O\left(n^{6}\right)}$, a quantifier-free formula of $\mathbf{R}$ which defines the set $V_{\text {sing } 1 \text {. }}$ 


\section{5. $C^{k}$-Locus, for $k \geq 2$}

The last subsection answers the problem of the determination of the $C^{k}$-smooth points of a semialgebraic set where $k=1$. Now, the case $k \geq 2$ is studied carefully.

Thanks to the following criterion, it is possible to get, for $k \geq 2$ fixed, an admissible algorithm for the points which are not $C^{k}$-smooth of a semialgebraic set.

Theorem 7 [PR]. Let $V$ be a closed semialgebraic set of $\mathbf{R}^{n}$. Let $f$ be the square of the Euclidean distance function from $V$ in $\mathbf{R}^{n}$ to $\mathbf{R}$ defined by

$$
f(x)=d^{2}(x, V)=\inf _{y \in V}\|x-y\|^{2} .
$$

Then, for $k \geq 2$, for each point, $x$, of $V$ :

$x$ is $C^{k}$-smooth in $V \Longleftrightarrow f$ is $C^{k}$-smooth in a neighborhood of $x$.

Proposition 3. Let $k$ be an integer number larger than or equal to 2. Let $V$ be a semialgebraic subset of $\mathbf{R}^{n}$ defined by a quantifier-free formula of $\mathbf{R}$ with coefficients in a subring $\mathbf{A}$ of $\mathbf{R}$ and with a degree at most $D(D \geq n)$.

Then, there exists a well-parallelizable arithmetic network on $\mathbf{A}$ with a sequential complexity $D^{n^{O(1)}}$ which builds a quantifier-free formula of $\mathbf{R}$ defining the semialgebraic set, $V_{\text {sing } k}$, of points of $V$ which are not $C^{k}$-smooth in $V$.

The proof provides the expected algorithm.

Proof. Theorem 7 allows for the detection of the points not $C^{k}$-smooth in a closed semialgebraic set. The set $V_{\text {sing } k}$ is defined by all the points of $V$ in the adherence of $\bar{V} \backslash V$ and by all the points of $V$ which are not $C^{k}$-smooth in $\bar{V}$.

In order to translate the two conditions into formulas, the Euclidean closure of the set $V$ is required.

$$
z \in \bar{V} \quad \Longleftrightarrow \quad \forall \varepsilon>0, \exists z^{\prime} \in V, \quad\left\|z-z^{\prime}\right\|^{2}<\varepsilon .
$$

This is a prenex formula of $\mathbf{R}$. It has $n$ free variables, and two quantifier blocks, respectively, of size 1 and $n$. The degree is at most $D+2$. A quantifier-free formula of $\mathbf{R}$ which defines $\bar{V}$ is obtained with sequential complexity $D^{O\left(n^{2}\right)}$ by the fast quantifier elimination algorithm (Theorem 4).

The fist condition becomes

$$
x \in \overline{(\bar{V} \backslash V)} \cap V \quad \Longleftrightarrow \quad \forall \varepsilon^{\prime}>0, \exists z \in \bar{V} \backslash V, \quad\|x, z\|^{2}<\varepsilon^{\prime} \quad \text { and } \quad x \in V .
$$

This is a prenex formula of $\mathbf{R}$ with two quantifier blocks, of size 1 and $n$ and with $n$ free variables. Its degree is at most $D^{O\left(n^{2}\right)}+2 D+3=D^{O\left(n^{2}\right)}$. The fast quantifier elimination algorithm (Theorem 4) constructs, with a sequential complexity $\left[D^{O\left(n^{2}\right)}\right]^{O\left(n^{2}\right)}=D^{O\left(n^{4}\right)}$, a quantifier-free formula of $\mathbf{R}$ which defines the set $\overline{(\bar{V} \backslash V)} \cap V$. 
Now, it remains to find the points of $V$ not $C^{k}$-smooth in $\bar{V}$. In order to construct those, Theorem 7 suggests to study the function, $f$, square of the Euclidean distance function to $\bar{V}$ :

$$
f(x)=d^{2}(x, \bar{V})=\inf _{y \in \bar{V}}\|x-y\|^{2}=\inf _{y \in V}\|x-y\|^{2} .
$$

The graph of $f$ is the following semialgebraic set:

$$
\begin{aligned}
\operatorname{graph}(f)= & \left\{(x, f(x)) \in \mathbf{R}^{n} \times \mathbf{R}:\left(\forall y \in V, f(x) \leq\|x-y\|^{2}\right)\right. \\
& \text { and } \left.\left(\forall \varepsilon^{\prime}>0, \exists z \in V,\|x-z\|^{2} \leq f(x)+\varepsilon^{\prime}\right)\right\} .
\end{aligned}
$$

This set is defined by a formula equivalent to a prenex formula of $\mathbf{R}$ with two quantifier blocks, of size $n+1, n$, and with $n+1$ free variables. The degree of this prenex formula is at most $2 D+5$. The set $\operatorname{graph}(f)$ is defined by a quantifier-free formula of $\mathbf{R}$ with sequential complexity $[2 D+5]^{O\left(n^{3}\right)}=D^{O\left(n^{3}\right)}$ by the fast quantifier elimination algorithm (Theorem 4).

The next step is the construction of the graph of the gradient of $f$. Lemma 3 and the fast quantifier elimination algorithm (Theorem 4) lead, with a sequential complexity $\left(D^{O\left(n^{3}\right)}\right)^{O\left(n^{2}\right)}=D^{O\left(n^{5}\right)}$, to a quantifier-free formula of $\mathbf{R}$ which defines the semialgebraic set graph $(\partial f)$ of $\mathbf{R}^{n} \times \mathbf{R}^{n}$ characterized by

$$
\begin{cases}\operatorname{graph}(\partial f) \cap\left(\{x\} \times \mathbf{R}^{n}\right)=\left\{\left(x, \frac{\partial f}{\partial x_{1}}(x), \ldots, \frac{\partial f}{\partial x_{n}}(x)\right)\right\} & \text { if } f \text { is differentiable and } \\ & \text { its gradient at } x \text { is } \\ \operatorname{graph}(\partial f) \cap\left(\{x\} \times \mathbf{R}^{n}\right)=\emptyset & \left(\frac{\partial f}{\partial x_{1}}(x), \ldots, \frac{\partial f}{\partial x_{n}}(x)\right), \\ \text { else. }\end{cases}
$$

Step by step, it is possible to get by the same method, with a sequential complexity $D^{n^{o(1)}}$, for each integer number $i$ smaller than or equal to $k$, a semialgebraic set of $\mathbf{R}^{n} \times \mathbf{R}^{n^{i}}$, $\operatorname{graph}\left(\partial^{i} f\right)$, such that for all $x$ of $V$ where $f$ is $i-1$ differentiable,

$$
\left\{\begin{aligned}
\operatorname{graph}\left(\partial^{i} f\right) \cap\left(\{x\} \times \mathbf{R}^{n^{i}}\right)=\{(x, & \left.\left.\left(\frac{\partial^{i} f}{\partial x_{j_{1}} \cdots \partial x_{j_{i}}}(x)\right)_{j_{1}, \ldots, j_{i}}\right)\right\} \\
& \text { if } f \text { is } i \text {-times differentiable at } x, \\
& \text { and if }\left(\left(\frac{\partial^{i} f}{\partial x_{j_{1}} \cdots \partial x_{j_{i}}}(x)\right)_{j_{1}, \ldots, j_{i}}\right) \text { are } \\
& \text { the partial derivatives of order } i \text { of } f \text { at } x \\
\operatorname{graph}\left(\partial^{i} f\right) \cap\left(\{x\} \times \mathbf{R}^{n^{i}}\right)=\emptyset & \text { else. }
\end{aligned}\right.
$$

Thus, the set of points of $V$ which are not $C^{k}$-smooth in $\bar{V}$ can be defined by

$$
\bar{V}_{\text {sing } k} \cap V=\bigcup_{i=1}^{k}\left\{x \in V: \operatorname{graph}\left(\partial^{i} f\right) \cap\left(\{x\} \times \mathbf{R}^{n^{i}}\right)=\emptyset\right\} \text {. }
$$


Then,

$$
\bar{V}_{\text {sing } k} \cap V=\bigcup_{i=1}^{k}\left\{x \in V: \forall t \in \mathbf{R}^{n^{i}},(x, t) \notin \operatorname{graph}\left(\partial^{i} f\right)\right\} .
$$

For every $i$ between 1 and $k$, the fast quantifier elimination algorithm (Theorem 4) applied to $\forall t \in \mathbf{R}^{n^{i}},(x, t) \notin \operatorname{graph}\left(\partial^{i} f\right)$, leads to an equivalent quantifier-free formula of $\mathbf{R}$ with a sequential complexity $\left[D^{n^{O(1)}}\right]^{O\left(n^{k}\right)}=D^{n^{O(1)}}$.

The set $V_{\text {sing } k}$ is $[\overline{(\bar{V} \backslash V)} \cap V] \cup\left[\bar{V}_{\text {sing } k} \cap V\right]$. Thus, it is defined by a quantifier-free formula of $\mathbf{R}$ with a sequential complexity $D^{n^{O(1)}}$.

\section{Stratification of Real Semialgebraic Sets}

\subsection{Admissible Stratifying Condition Over $\mathbf{R}$}

The admissibility over $\mathbf{R}$ of a stratifying condition on $\mathbf{R}$ is introduced in this section. An elementary but useful criterion of admissibility over $\mathbf{R}$ is given and is used in the proof of admissibility of the Whitney conditions or the (C)-condition.

Definition 20. A stratifying condition of order $k$ is admissible over $\mathbf{R}$ if and only if, for each pair, $(X, Y)$, of disjoint $C^{k}$-smooth semialgebraic sets of $\mathbf{R}^{n}$, each defined by a quantifier-free formula of $\mathbf{R}$ of degree at most $D(D \geq n)$ and such that $Y \subset \bar{X}$, there exists a well-parallelizable arithmetic network with a sequential complexity $D^{n^{o(1)}}$ which computes a quantifier-free formula of $\mathbf{R}$ defining the set of points, $y$, of $Y$ where the stratifying condition fails at $(X, Y, y)$.

Remark. This is just the admissibility of an algorithm (Definition 18) adapted to stratifying conditions on $\mathbf{R}$.

Lemma 5. Let $m$ be a fixed integer number. Let $(I)$ be a stratifying condition on $\mathbf{R}$ of order $k$ such that:

for each pair, $(X, Y)$, of disjoint $C^{k}$-smooth semialgebraic sets of $\mathbf{R}^{n}$, each defined by a quantifier-free formula of $\mathbf{R}$ of degree at most $D(D \geq n)$ and such that $Y \subset \bar{X}$, there exists a well-parallelizable arithmetic network with a sequential complexity $D^{n^{o(1)}}$ which computes a prenex formula of $\mathbf{R}$ of degree at most $D^{n^{o(1)}}$ and with at most $m$ quantifier blocks, with at most $n^{O(1)}$ variables in each of them and which defines the set of points, $y$, of $Y$ where the stratifying condition fails at $(X, Y, y)$.

Then (I) is an admissible condition of order $k$ over $\mathbf{R}$.

Proof. The fast quantifier elimination (Theorem 4) immediately gives the result. 


\subsection{The Canonical Stratification Algorithm}

This subsection is devoted to the complexity estimate of the computation of the canonical $C^{k}$-stratification which satisfies an admissible condition over $\mathbf{R}$.

Let $(I)$ be an admissible stratifying condition of order $k^{\prime}$ over $\mathbf{R}$.

Let $k$ be a fixed integer number larger than or equal to $k^{\prime}$.

Let $V$ be a semialgebraic set of $\mathbf{R}^{n}$ defined by a quantifier-free formula of $\mathbf{R}$ of degree at most $D(D \geq n)$.

The canonical (I)-regular $C^{k}$-stratification of $V$ is defined by a filtration by closed subsets of $V$ as shown in the proof of Theorem 3:

$$
F_{0}=V \supset F_{1} \supset \cdots \supset F_{i} \supset \cdots \supset F_{l}=\emptyset
$$

defined by

$$
\left\{\begin{aligned}
F_{1}=F_{0, \text { sing } k}= & V_{\text {sing } k} \text { and, for } i=2, \ldots, l \\
F_{i}=F_{i-1, \text { sing } k} \cup & {\left[\overline{\bigcup_{C \in \mathcal{S}_{i-1}} I\left(C,\left(F_{i-1, \text { reg } k} \cap \bar{C}\right)_{\text {reg } k}\right)} \cap V\right] } \\
\cup & {\left[\bigcup_{C \in \mathcal{S}_{i-1}}\left(\bar{C} \cap \overline{\left(F_{i-1} \backslash \bar{C}\right)} \cap V\right)\right] }
\end{aligned}\right.
$$

The strata of the canonical (I)-regular $C^{k}$-stratification $\mathcal{S}_{l}$ of $V$ are the connected components of the $F_{j} \backslash F_{j+1}$ for $j$ from 0 to $l-1$.

The necessary tools for this construction are:

(i) An empty-set test for a semialgebraic set. Only Theorem 5 is needed.

(ii) An algorithm of determination of the $C^{k}$-locus of a semialgebraic set. It is done by an admissible algorithm (Proposition 2 if $k=1$ and Proposition 3 for the other values of $k$ ).

(iii) An algorithm of determination of sets $I(X, Y)$ where condition $(I)$ fails. It is done by the admissibility of Condition $(I)$ over $\mathbf{R}$.

(iv) An algorithm of determination of the Euclidean closure of a semialgebraic set of $\mathbf{R}^{n}$. But, the Euclidean closure of a set $A$ is the set of points where the following formula is true: $\forall \varepsilon>0, \exists y \in A,\|x-y\|^{2}<\varepsilon$. The fast quantifier elimination (Theorem 4) leads to the expected admissible algorithm.

\section{* Sequential Complexity of this Algorithm}

The step of the computation of the $i$ th element $F_{i}$ of the associated filtration needs:

(i) One use of the empty-set test (Theorem 5) which has a sequential complexity singly exponential in $n$.

(ii) One computation of the $C^{k}$-locus in order to get $F_{i-1, \operatorname{sing} k}$ which also has a sequential complexity singly exponential in $n$.

(iii) $\#\left(\mathcal{S}_{i-1}^{*}\right)$ independent computations of $\bar{C}$, each of them with a sequential complexity singly exponential in $n$. 
(iv) \#( $\left.\mathcal{S}_{i-1}\right)$ independent applications of the algorithm of $C^{k}$-locus determination to build the sets $\left(F_{i-1, \text { reg } k} \cap \bar{C}\right)_{\text {reg } k}$. Each of them has a sequential complexity singly exponential in $n$.

(v) $\#\left(\mathcal{S}_{i-1}\right)$ independent applications of the algorithm of the determination of the set where Condition ( $I$ ) fails for two semialgebraic sets. By the admissibility over $\mathbf{R}$ of Condition $(I)$, each computation has a sequential complexity singly exponential in $n$.

(vi) One computation of Euclidean closure of a semialgebraic set which also has a sequential complexity singly exponential in $n$.

(vii) $\#\left(\mathcal{S}_{i-1}\right)$ independent computations of $\bar{C} \cap \overline{\left(F_{i-1} \backslash \bar{C}\right)} \cap V$. Each of them has a sequential complexity singly exponential in $n$

(viii) One application of Theorem 6 in order to construct new strata.

The total sequential complexity of this algorithm is

$$
\left(\left(D^{n^{O(1)}}\right)^{\cdots{ }^{l \text { times }} \cdots}\right)^{n^{O(1)}}=D^{n^{o(l)}} .
$$

It is doubly exponential in the depth of filtration associated to the canonical ( $I$ )-regular $C^{k}$-stratification of the input semialgebraic set.

Thus, the following result has just been proved.

Theorem 8. Let $(I)$ be an admissible stratifying condition of order $k^{\prime}$ over $\mathbf{R}$.

Let $k$ be a fixed integer number larger than or equal to $k^{\prime}$.

Let $V$ be a semialgebraic set of $\mathbf{R}^{n}$ defined by a quantifier-free formula of $\mathbf{R}$ of degree at most $D(D \geq n)$.

Let $l$ be the depth of the filtration associated to the canonical (I)-regular $C^{k}$ stratification of $V$.

Then, there exists a well-parallelizable arithmetic network with a sequential complexity $D^{n^{o(l)}}$ which computes quantifier-free formulas of $\mathbf{R}$ defining every stratum of the canonical (I)-regular $C^{k}$-stratification of $V$ and also each element of the associated filtration.

The proof provides the expected algorithm.

Remark. The previous result applied to Conditions $\left(\emptyset_{k}\right)$ leads to canonical $C^{k}$-stratification algorithms for semialgebraic sets.

\subsection{Admissibility Over $\mathbf{R}$ of the Whitney Conditions}

The aim of this part is to show the admissibility over $\mathbf{R}$ of the Whitney conditions and to apply this result in order to get algorithms for Whitney stratification computation. 
Proposition 4. The Whitney conditions (a) and (b) are both admissible stratifying conditions of order 1 over $\mathbf{R}$.

Proof. Let $X$ and $Y$ be two disjoint $C^{1}$-smooth semialgebraic sets of $\mathbf{R}^{n}$ such that $Y \subset \bar{X}$, each defined by a quantifier-free formula of $\mathbf{R}$ of degree at most $D(n \leq D)$.

Translating the two Whitney conditions into formulas of $\mathbf{R}$ and applying the admissibility over $\mathbf{R}$ criterion (Lemma 5) is enough to get the admissibility.

* The Whitney Condition (a)

Let

$$
F_{a}(X)=\left\{(x, T) \in \mathbf{R}^{n} \times\left(\bigcup_{k=0}^{n} \mathbf{G}_{n, k}(\mathbf{R})\right): x \in X \text { and } T=T_{x}^{X}\right\} .
$$

Let $y$ be a point of $Y$. The Whitney condition (a) holds at $(X, Y, y)$ if and only if

$$
\text { for all } \tau \text { of } \bigcup_{k=0}^{n} \mathbf{G}_{n, k}(\mathbf{R}), \quad(y, \tau) \in \overline{F_{a}(X)} \quad \Longrightarrow \quad \tau \supset T_{y}^{Y} .
$$

According to Lemma 4, the Grassmannian $\mathbf{G}_{n, k}(\mathbf{R})$ is represented by the algebraic subset of $\mathbf{M}_{n, n}(\mathbf{R}) \simeq \mathbf{R}^{n^{2}}$ defined by

$$
\mathbf{G}_{n, k}(\mathbf{R})=\left\{A \in \mathbf{M}_{n, n}(\mathbf{R}): A={ }^{t} A, A^{2}=A, \operatorname{trace}(A)=k\right\} .
$$

Hence, the $\left(\bigcup_{k=0}^{n} \mathbf{G}_{n, k}(\mathbf{R})\right)$ is represented by the set of all the matrices, $A$, of $\mathbf{M}_{n, n}(\mathbf{R})$ such that $A={ }^{t} A$ and $A^{2}=A$.

Thus, by Lemma 4

$$
\begin{aligned}
F_{a}(X)=\{ & \left(x,\left(a_{i, j}\right)_{i, j}\right) \in \mathbf{R}^{n} \times \mathbf{M}_{n, n}(\mathbf{R}):(x \in X) \text { and } \\
& {\left[\forall\left(t_{1}, \ldots, t_{n}\right) \in T_{x}^{X}, \sum_{i=1}^{n} a_{i, j} t_{i}=t_{j} \text { and }\left(a_{1, j}, \ldots, a_{n, j}\right) \in T_{x}^{X}\right.} \\
& \quad \text { for all } j=1, \ldots, n]\} .
\end{aligned}
$$

Lemma 2 allows to translate the tangent space $T_{x}^{X}$ into a formula of $\mathbf{R}$ ( $x$ is considered as a parameter)

$$
\forall \eta>0, \forall \varepsilon>0, \exists u, v \in B(x, \eta) \cap X, \exists \lambda \in \mathbf{R}, \quad\|t-\lambda(u-v)\|^{2} \leq \varepsilon .
$$

This formula has $2 n$ free variables. The fast quantifier elimination algorithm (Theorem 4 ) with this formula as an input gives an equivalent quantifier-free formula of $\mathbf{R}$ with a sequential complexity $D^{O\left(n^{2}\right)}$.

The same arguments with $Y$ instead of $X$, lead to a quantifier-free formula of $\mathbf{R}$ which defines the tangent bundle $T^{Y}$ with a sequential complexity $D^{O\left(n^{2}\right)}$. Let $W_{a}(X, Y)$ the set of $y \in Y$ where the Whitney condition (a) fails at $(X, Y, y)$.

$$
W_{a}(X, Y)=\left\{y \in Y: \exists \tau \in\left(\bigcup_{k=0}^{n} \mathbf{G}_{n, k}(\mathbf{R})\right),(y, \tau) \in \overline{F_{a}(X)} \text { and } T_{y}^{Y} \not \subset \tau\right\} \text {, }
$$


i.e.,

$$
W_{a}(X, Y)=\left\{y \in Y: \exists \tau \in\left(\bigcup_{k=0}^{n} \mathbf{G}_{n, k}(\mathbf{R})\right), \exists t \in T_{y}^{Y},(y, \tau) \in \overline{F_{a}(X)} \text { and } t \notin \tau\right\} .
$$

The translation of the Euclidean closure of $F_{a}(X)$ is required to express the condition $(y, \tau) \in \overline{F_{a}(X)}$. This is just the set of all the pairs, $\left(y,\left(a_{i, j}\right)_{i, j}\right)$, of $\mathbf{R}^{n} \times \mathbf{M}_{n, n}(\mathbf{R})$ such that

$$
\forall \varepsilon>0, \exists\left(x,\left(a_{i, j}^{\prime}\right)_{i, j}\right) \in F_{a}(X), \quad\|y-x\|^{2}+\sum_{i, j}\left(a_{i, j}-a_{i, j}^{\prime}\right)^{2}<\varepsilon .
$$

This is equivalent to

$$
\begin{gathered}
\forall \varepsilon>0, \exists\left(x,\left(a_{i, j}^{\prime}\right)_{i, j}\right) \in \mathbf{R}^{n} \times \mathbf{M}_{n, n}(\mathbf{R}), \forall\left(t_{1}^{\prime}, \ldots, t_{n}^{\prime}\right) \in T_{x}^{X},(x \in X) \text { and } \\
{\left[\sum_{i=1}^{n} a_{i, j}^{\prime} t_{i}^{\prime}=t_{j}^{\prime} \text { and }\left(a_{1, j}^{\prime}, \ldots, a_{n, j}^{\prime}\right) \in T_{x}^{X} \text { for all } j=1, \ldots, n\right]}
\end{gathered}
$$

and

$$
\left(\|y-x\|^{2}+\sum_{i, j}\left(a_{i, j}-a_{i, j}^{\prime}\right)^{2}<\varepsilon\right) .
$$

This is equivalent to a prenex formula of $\mathbf{R}$ with three quantifier blocks, respectively, of size $1, n+n^{2}$, and $n$, with $n+n^{2}$ free variables. Its degree is at most $(n+1) D^{O\left(n^{2}\right)}+$ $2 n+D+3=D^{O\left(n^{2}\right)}$.

In the union of all Grassmannians $\bigcup_{k=0}^{n} \mathbf{G}_{n, k}(\mathbf{R})$, a vector space $\tau$ is represented by the matrix $A$ of its orthogonal projection in the canonical bases. Then, the condition $t \notin \tau$ becomes $A t \neq t$. But, $\bigcup_{k=0}^{n} \mathbf{G}_{n, k}(\mathbf{R})$ is closed in $\mathbf{M}_{n, n}(\mathbf{R})$, hence

$$
W_{a}(X, Y)=\left\{y \in Y: \exists A \in \mathbf{M}_{n, n}(\mathbf{R}), \exists t \in T_{y}^{Y},(y, A) \in \overline{F_{a}(X)} \text { and } A t \neq t\right\} .
$$

Thus, the set $W_{a}(X, Y)$ can be defined by a prenex formula of $\mathbf{R}$ with at most five quantifier blocks, each of them of size at most $n^{2}+n$ and with $n$ free variables. Its degree is at most $D^{O\left(n^{2}\right)}$. The admissibility over $\mathbf{R}$ criterion (Lemma 5) shows the admissibility over $\mathbf{R}$ of Whitney condition (a).

\section{* The Whitney Condition (b)}

Let

$$
F_{b}(X, Y)=\left\{(x, T, y, t) \in F_{a}(X) \times \mathbf{R}^{n} \times \mathbf{R}^{n}: y \in Y \text { and } t \in \widehat{x y}\right\} .
$$

The Whitney condition (b) holds at $(X, Y, y)$ if and only if:

$$
\begin{aligned}
& \text { for every } \tau \text { of } \bigcup_{k=0}^{n} \mathbf{G}_{n, k}(\mathbf{R}) \text { and for every } \delta \text { in } \mathbf{R}^{n}, \\
& \qquad(y, \tau, y, \delta) \in \overline{F_{b}(X, Y)} \Longrightarrow \delta \in \tau .
\end{aligned}
$$

* Construction of the Set $F_{b}(X, Y)$

The set $F_{b}(X, Y)$ is the set of all the $(y, T, x, t)$ of $\mathbf{R}^{n} \times \mathbf{M}_{n, n}(\mathbf{R}) \times \mathbf{R}^{n} \times \mathbf{R}^{n}$ such that

$$
x \in X \quad \text { and } \quad T=T_{x}^{X} \quad \text { and } \quad y \in Y \quad \text { and } \quad(\exists \lambda \in \mathbf{R}, t=\lambda(x-y)) .
$$


The set $\overline{F_{b}(X, Y)}$ contains $(y, T, x, t)$ if and only if:

$$
\begin{gathered}
\forall \varepsilon>0, \exists\left(y^{\prime}, T^{\prime}, x^{\prime}, t^{\prime}\right) \in F_{b}(X, Y), \\
\left\|y^{\prime}-y\right\|^{2}+\left\|T^{\prime}-T\right\|^{2}+\left\|x^{\prime}-x\right\|^{2}+\left\|t^{\prime}-t\right\|^{2} \leq \varepsilon .
\end{gathered}
$$

This equivalent to

$$
\begin{aligned}
& \forall \varepsilon>0, \exists y^{\prime} \in \mathbf{R}^{n}, \exists T^{\prime} \in \mathbf{M}_{n, n}(\mathbf{R}), \exists x^{\prime} \in \mathbf{R}^{n}, \exists t^{\prime} \in \mathbf{R}^{n}, \exists \lambda \in \mathbf{R}, \\
& x^{\prime} \in X \quad \text { and } \quad T^{\prime}=T_{x^{\prime}}^{X} \text { and } \quad y^{\prime} \in Y \quad \text { and } \quad t^{\prime}=\lambda\left(x^{\prime}-y^{\prime}\right)
\end{aligned}
$$

and

$$
\left\|y^{\prime}-y\right\|^{2}+\left\|T^{\prime}-T\right\|^{2}+\left\|x^{\prime}-x\right\|^{2}+\left\|t^{\prime}-t\right\|^{2} \leq \varepsilon .
$$

This is a prenex formula of $\mathbf{R}$ with two quantifier blocks, respectively, of size $1, n^{2}+$ $3 n+1$. It has $n^{2}+3 n$ free variables. Its degree is at most $D^{O\left(n^{2}\right)}+2 D+5=D^{O\left(n^{2}\right)}$.

Let $W(X, Y)$ be the set of points $y$ of $Y$ where the Whitney condition (b) does not hold at $(X, Y, y)$. Then,

$$
W(X, Y)=\left\{y \in Y: \exists(A, \delta) \in \mathbf{M}_{n, n}(\mathbf{R}) \times \mathbf{R}^{n},(y, A, y, \delta) \in \overline{F_{b}(X, Y)} \text { and } A \delta \neq \delta\right\}
$$

The two formulas are combined together in order to get a prenex formula of $\mathbf{R}$ which defines $W(X, Y)$. The formula has at most three quantifier blocks, each of them of size at most $n^{2}+3 n+1$ and with $n$ free variables. Its degree is at most $D^{O\left(n^{2}\right)}$. The admissibility over $\mathbf{R}$ criterion (Lemma 5) leads to the admissibility over $\mathbf{R}$ of the Whitney condition (b).

Theorem 8 applied to the Whitney conditions proves the following result:

Theorem 9. Let $k$ be a positive integer number:

Let $V$ be a semialgebraic set of $\mathbf{R}^{n}$ defined by a quantifier-free formula of $\mathbf{R}$, with degree at most $D(D \geq n)$.

Let $l_{a}\left(\right.$ resp., $\left.l_{b}\right)$ be the depth of the filtration associated to the canonical Whitney (a)-regular (resp., Whitney (b)-regular) $C^{k}$-stratification of $V$.

Then, there exist two well-parallelizable arithmetic networks such that:

(i) one of them builds, with a sequential complexity $D^{n^{o(l a)}}$, quantifier-free formulas of $\mathbf{R}$ which define all the strata of the canonical Whitney $(a)$-regular $C^{k}$ stratification of $V$ and also each element of the associated filtration; and

(ii) the other builds, with a sequential complexity $D^{n^{o\left(l_{b}\right)}}$, quantifier-free formulas of $\mathbf{R}$ which define all the strata of the canonical Whitney $(b)$-regular $C^{k}$-stratification of $V$ and also each element of the associated filtration.

The proof provides the expected algorithm. 


\subsection{Condition $(C)$}

Proposition 5. Condition $(C)$ (see Definitions 10 and 11) with functions equal to the square of the Euclidean distances to the strata is an admissible stratifying condition over $\mathbf{R}$ of order 1.

Proof. In order to prove the result by the admissibility over $\mathbf{R}$ criterion (Lemma 5), Condition $(\mathrm{C})$ is translated into formulas of $\mathbf{R}$.

Let $X$ and $Y$ be two disjoint $C^{1}$-smooth semialgebraic sets of $\mathbf{R}^{n}$ such that $Y \subset \bar{X}$, each defined by a quantifier-free formula of $\mathbf{R}$ of degree at most $D(n \leq D)$. Let $f$ be the function defined by

$$
\begin{aligned}
f: \mathbf{R}^{n} & \longrightarrow \mathbf{R}^{+}, \\
x & \longmapsto d^{2}(x, Y)=\inf _{y \in Y}\|x-y\|^{2} .
\end{aligned}
$$

Condition (C) uses the vectors spaces Ker $\partial_{x}\left(\left.f\right|_{X}\right)$ and $\operatorname{Ker} \partial_{y}\left(\left.f\right|_{Y}\right)$ (see Definitions 10 and 11).

$$
\operatorname{Ker} \partial_{x}\left(\left.f\right|_{X}\right)=\left(\operatorname{grad}_{x} f\right)^{\perp} \cap T_{x}^{X} \quad \text { and } \quad \operatorname{Ker} \partial_{x}\left(\left.f\right|_{Y}\right)=\left(\operatorname{grad}_{y} f\right)^{\perp} \cap T_{y}^{Y} .
$$

Those objects must be translated into formula of $\mathbf{R}$.

* Translation of $f$ (see the proof of Proposition 3)

The graph of $f$ is the set of all the points $(x, f(x))$ such that

$$
\left(\forall y \in Y, f(x) \leq d^{2}(x, y)\right) \quad \text { and } \quad\left(\forall \varepsilon^{\prime}>0, \exists z \in Y,\|x-z\|^{2} \leq f(x)+\varepsilon^{\prime}\right) .
$$

After having combined together the quantifier blocks, this formula becomes a prenex formula of $\mathbf{R}$ with two quantifier blocks, respectively, of size $n+1, n$, and with $n+1$ free variables. Its degree is at most $2 D+5$. Renegar's version of the Tarski-Seidenberg principle (Theorem 4) provides an equivalent quantifier-free formula with a sequential complexity $D^{O\left(n^{3}\right)}$.

* Translation of the Gradient $\operatorname{grad}_{x} f$ (see the proof of Proposition 3)

Lemma 3 and the fast quantifier elimination algorithm (Theorem 4) lead, with a sequential complexity $\left[D^{O\left(n^{3}\right)}\right]^{O\left(n^{2}\right)}=D^{O\left(n^{5}\right)}$, to a quantifier-free formula of $\mathbf{R}$ which defines a subset graph $(\operatorname{grad} f)$ of $\mathbf{R}^{n} \times \mathbf{R}^{n}$ such that

$$
\begin{cases}\operatorname{graph}(\operatorname{grad} f) \cap\left(\{x\} \times \mathbf{R}^{n}\right) & \\ =\left\{\left(x, \frac{\partial f}{\partial x_{1}}(x), \ldots, \frac{\partial f}{\partial x_{n}}(x)\right)\right\} & \text { if } f \text { is differentiable } \\ & \text { at } x \text { and its gradient at } x \text { is } \\ \operatorname{graph}(\operatorname{grad} f) \cap\left(\{x\} \times \mathbf{R}^{n}\right)=\emptyset & \left(\frac{\partial f}{\partial x_{1}}(x), \ldots, \frac{\partial f}{\partial x_{n}}(x)\right),\end{cases}
$$

* Translation of $T_{x}^{X}$ and $T_{y}^{Y}$

The same construction is done during the proof of Proposition 4. The required sequential complexity is $D^{O\left(n^{2}\right)}$. 
* Translation of the Kernel of the Differential of $\left.f\right|_{X}$ at $x$, Ker $\left.\partial_{x} f\right|_{X}$ Since $\left(\operatorname{grad}_{x} f\right)^{\perp}=\left\{t \in \mathbf{R}^{n}: \operatorname{grad}_{x} f{ }^{t} t=0\right\}$, the set Ker $\left.\partial_{x} f\right|_{X}$ is characterized by

$\operatorname{Ker} \partial_{x}\left(\left.f\right|_{X}\right)=\left(\operatorname{grad}_{x} f\right)^{\perp} \cap T_{x}^{X}=\left\{t \in \mathbf{R}^{n}: t \in T_{x}^{X}\right.$ and $\left.\operatorname{grad}_{x} f{ }^{t} t=0\right\}$.

Then, the set $\operatorname{Ker} \partial_{x}\left(\left.f\right|_{X}\right)$ is the set of all the vectors $t$ of $\mathbf{R}^{n}$ such that:

$$
t \in T_{x}^{X} \quad \text { and } \quad\left(\forall t^{\prime} \in \mathbf{R}^{n}\left(x, t^{\prime}\right) \in \operatorname{graph}(\operatorname{grad} f) \Longrightarrow t^{\prime} \cdot{ }^{t} t=0\right) .
$$

This is equivalent to a prenex formula of $\mathbf{R}$ with a single quantifier block which contains $n$ real variables. This formula has $2 n$ free variables ( $x$ is seen as a parameter). Its degree is at most $D^{O\left(n^{2}\right)}+D^{O\left(n^{5}\right)}+2=D^{O\left(n^{5}\right)}$. The fast quantifier elimination algorithm (Theorem 4), leads, with a sequential complexity $\left[D^{O\left(n^{5}\right)}\right]^{O\left(n^{2}\right)}=D^{O\left(n^{7}\right)}$, to an equivalent quantifier-free formula of $\mathbf{R}$. The same thing can be done with $Y$ instead of $X$.

* Translation of Condition (C)

Let

$$
F(X)=\left\{(x, T) \in \mathbf{R}^{n} \times\left(\bigcup_{k=0}^{n} \mathbf{G}_{n, k}(\mathbf{R})\right): x \in X \text { and } T=\operatorname{Ker} \partial_{x}\left(\left.f\right|_{X}\right)\right\} .
$$

Condition (C) holds at $(X, Y, y)$ if and only if

$$
\forall \tau \in \bigcup_{k=0}^{n} \mathbf{G}_{n, k}(\mathbf{R}), \quad\left((y, \tau) \in \overline{F(X)} \quad \Longrightarrow \quad \operatorname{Ker} \partial_{y}\left(\left.f\right|_{Y}\right) \subset \tau\right) .
$$

According to Lemma 4 and the chosen representation of the Grassmannian, the condition $\operatorname{Ker} \partial_{y}\left(\left.f\right|_{Y}\right) \subset \tau$ becomes: $\forall t \in \operatorname{Ker} \partial_{y}\left(\left.f\right|_{Y}\right), A t=t$. By the translation of the Euclidean closure of the set $F(X)$ and by Lemma 4 , the set $\overline{F(X)}$ is the set of all the pairs $\left(x,\left(a_{i, j}^{\prime}\right)_{i, j}\right)$ of $\mathbf{R}^{n} \times \mathbf{M}_{n, n}(\mathbf{R})$ such that

$$
\forall \varepsilon>0, \exists\left(x^{\prime},\left(a_{i, j}^{\prime}\right)_{i, j}\right) \in \mathbf{R}^{n} \times \mathbf{M}_{n, n}(\mathbf{R}), \forall t^{\prime} \in \operatorname{Ker} \partial_{x^{\prime}}\left(\left.f\right|_{X}\right), \quad\left(x^{\prime} \in X\right)
$$

and

$$
\left[\sum_{i=1}^{n} a_{i, j}^{\prime} t_{i}^{\prime}=t_{j}^{\prime} \text { and }\left(a_{1, j}^{\prime}, \ldots, a_{n, j}^{\prime}\right) \in \operatorname{Ker} \partial_{x^{\prime}}\left(\left.f\right|_{X}\right) \text { for all } j=1, \ldots, n\right]
$$

and

$$
\left(\left\|x^{\prime}-x\right\|^{2}+\sum_{i, j}\left(a_{i, j}-a_{i, j}^{\prime}\right)^{2}<\varepsilon\right) .
$$

where $t_{i}^{\prime}$ represents $i$ th coordinate of the vector $t^{\prime}$.

Then, the set $\overline{F(X)}$ is defined by a prenex formula of $\mathbf{R}$ with three quantifier blocks, respectively, of size $1, n+n^{2}, n$, with $n+n^{2}$ free variables. Its degree is at most $D^{O\left(n^{7}\right)}$. Let $C(X, Y)$ be the set of all the points, $y$, of $Y$ where Condition (C) with functions equal to the square of the Euclidean distances to the strata fails at $(X, Y, y)$. This set is defined by

$$
\exists A \in \mathbf{M}_{n, n}(\mathbf{R}), \exists t \in \operatorname{Ker} \partial_{y}\left(\left.f\right|_{Y}\right), \quad(y, A) \in \overline{F(X)} \quad \text { and } \quad A t \neq t .
$$


In order to get a formula for $C(X, Y)$, the two last formulas are combined together. This leads to a prenex formula of $\mathbf{R}$ with at most four quantifier blocks, each of them of size at most $n^{2}+n$. Its degree is at most $D^{O\left(n^{7}\right)}$. The admissibility over $\mathbf{R}$ criterion (Lemma 5) proves the admissibility over $\mathbf{R}$ of Condition (C) with functions equal to the square of the Euclidean distances to the strata.

Theorem 8 applied to Condition (C) with functions equal to the square of the Euclidean distances to the strata proves the following result:

Theorem 10. Let $k$ be a positive integer number:

Let $V$ be a semialgebraic set of $\mathbf{R}^{n}$ defined by a quantifier-free formula of $\mathbf{R}$, with degree at most $D(D \geq n)$.

Let $l$ be the depth of the filtration associated to the canonical $C^{k}$-stratification $(C)$ regular with functions equal to the square of the Euclidean distances to the strata of $V$.

Then, there exists a well-parallelizable arithmetic network which computes, with a sequential complexity $D^{n^{o(t)}}$, quantifier-free formulas of $\mathbf{R}$ defining all the strata of the canonical $C^{k}$-stratification $(C)$-regular with functions equal to the square of the Euclidean distances to the strata of $V$ and all elements of the associated filtration also.

The proof provides the expected algorithm.

\section{Stratifications of Complex Constructible Sets}

The methods used in the real semialgebraic case can be extended to the complex constructive case. This is the aim of this section.

\subsection{Admissibility Over $\mathbf{R}[i]$ :}

Strata are defined as connected components. In the case of constructible sets of $\mathbf{C}^{n}$, connected components are the irreducible components of the Zariski closure. No known well-parallelizable algorithm can compute them with a reasonable complexity (singly exponential in the dimension of the surrounding affine space). A solution is to identify $\mathbf{C}$ with $\mathbf{R}^{2}$ and to use the semialgebraic algorithm.

Definition 21. A stratifying condition on $\mathbf{C}$ is admissible over $\mathbf{R}[i]$ if and only if for each pair, $(X, Y)$ of disjoint $C^{\infty}$ constructible sets of $\mathbf{C}^{n}$ such that:

(i) $X$ and $Y$ are defined, after the identification of $\mathbf{C}^{n}$ and $\mathbf{R}^{2 n}$ by the map $\left(z_{i}\right)_{i=1, \ldots, n} \mapsto$ $\left(\operatorname{Re} z_{i}, \operatorname{Im} z_{i}\right)_{i=1, \ldots, n}$, by a quantifier-free formula of $\mathbf{R}$ of degree at most $D$ $(D \geq n) ;$ and

(ii) $Y \subset \bar{X}$; 
there exists a well-parallelizable arithmetic network on $\mathbf{R}$ which constructs, with a sequential complexity $D^{n^{O(1)}}$, a quantifier-free formula of $\mathbf{R}$ defining the image by the identification of $\mathbf{C}^{n}$ and of $\mathbf{R}^{2 n}$ of the set of all the points, $y$, of $Y$ where the stratifying condition fails at $(X, Y, y)$.

Remarks. (i) All the algorithms built here use only semialgebraic sets of $\mathbf{R}^{2 n}$. The strata are constructible sets of $\mathbf{C}^{n}$. Thus, it is necessary to be sure that the used semialgebraic sets of $\mathbf{R}^{2 n}$ are the images of constructible sets of $\mathbf{C}^{n}$ by the ordinary identification. But that is required by Definition 7 of stratifying condition on $\mathbf{C}$ for the sets where the condition does not hold.

(ii) For constructible sets of $\mathbf{C}^{n}, C^{1}$ and $C^{\infty}$ are equivalent. The $C^{k}$-regularities are useless here. Thus, only $C^{\infty}$-stratifications, i.e., stratifications, are used.

Like the real case, the following elementary but useful criterion implies the admissibility over $\mathbf{R}[i]$ of stratifying conditions.

Lemma 6. Let $m$ be a fixed integer number. Let $(I)$ be a stratifying condition on $\mathbf{C}$ such that for each pair, $(X, Y)$, of disjoint $C^{\infty}$, constructible sets of $\mathbf{C}^{n}$ such that:

(i) $X$ and $Y$ are defined, after the identification of $\mathbf{C}^{n}$ and $\mathbf{R}^{2 n}$ by the map $\left(z_{i}\right)_{i=1, \ldots, n} \mapsto$ $\left(\operatorname{Re} z_{i}, \operatorname{Im} z_{i}\right)_{i=1, \ldots, n}$, by a quantifier-free formula of $\mathbf{R}$ and of degree at most $D$ $(D \geq n)$; and

(ii) $Y \subset \bar{X}$;

there exists a well-parallelizable arithmetic network on $\mathbf{R}$ which constructs, with a sequential complexity $D^{n^{o(1)}}$, a prenex formula of $\mathbf{R}$ of degree at most $D^{n^{o(1)}}$ and with at most $m$ quantifier blocks, each of them of size at most $n^{O(1)}$ and which defines the image by the identification of $\mathbf{C}^{n}$ and of $\mathbf{R}^{2 n}$ of the set of all the points, $y$, of $Y$ where the stratifying condition (I) fails at $(X, Y, y)$.

Then $(I)$ is an admissible stratifying condition over $\mathbf{R}[i]$.

Proof. The fast quantifier elimination algorithm (Theorem 4) leads immediately to the result.

\subsection{The Canonical Stratification Algorithm}

Let $i^{\mathbf{R}}$ be the map defined by

$$
\begin{aligned}
\mathbf{C}^{n} & \longrightarrow \mathbf{R}^{2 n}, \\
\left(z_{i}\right)_{i=1, \ldots, n} & \longmapsto\left(\operatorname{Re} z_{i}, \operatorname{Im} z_{i}\right)_{i=1, \ldots, n} .
\end{aligned}
$$

Let $i^{\mathbf{C}}$ be its inverse. Let $A^{\mathbf{R}}$ be the image by $i^{\mathbf{R}}$ of a set $A$ of $\mathbf{C}^{n}$ and let $B^{\mathbf{C}}$ be the image by $i^{\mathbf{C}}$ of a set $B$ of $\mathbf{R}^{2 n}$.

Remarks. Let $A$ be a constructible set of $\mathbf{C}^{n}$. Then:

(i) $\left(A_{\text {sing }}\right)^{\mathbf{R}}=\left(A_{\text {sing } 1}\right)^{\mathbf{R}}=\left(A^{\mathbf{R}}\right)_{\text {sing } 1}$.

(ii) $(\bar{A})^{\mathbf{R}}=\overline{A^{\mathbf{R}}}$. 
(iii) The images by $i^{\mathbf{R}}$ of the connected components of $A$ are the connected components of the image of $A$ by $i^{\mathbf{R}}$.

Let $(I)$ be an admissible stratifying condition over $\mathbf{R}[i]$. Let $V$ be a constructible set of $\mathbf{C}^{n}$ of which the image by $i^{\mathbf{R}}$ is a semialgebraic set, $V^{\mathbf{R}}$, of $\mathbf{R}^{2 n}$ defined by a quantifier-free formula of $\mathbf{R}$ of degree at most $D(D \geq n)$.

Theorem 3 leads to the following computation algorithm for the canonical ( $I$ )-regular stratification of $V$. Here again, the notations of the proof of Theorem 3 are used.

* Initial condition: $F_{0}^{\mathbf{R}}=V^{\mathbf{R}}$.

The step $i=1$ :

$*$ Test by means of Theorem 5 whether $V^{\mathbf{R}}=\emptyset$.

$*$ If $V^{\mathbf{R}}=\emptyset, l=0$, then the filtration computation is completed and $\mathcal{S}_{0}^{\mathbf{R}}=\emptyset$ is the expected stratification.

* Otherwise, $F_{1}^{\mathbf{R}}$ is built by $F_{1}^{\mathbf{R}}=\left(F_{0, \text { sing }}\right)^{\mathbf{R}}=\left(F_{0}^{\mathbf{R}}\right)_{\operatorname{sing} 1}=V_{\text {sing } 1}^{\mathbf{R}}$. It can be done by Proposition 2.

* Computation of the set, $\mathcal{S}_{1}^{\mathbf{R}}$, of all connected components of $F_{0}^{\mathbf{R}} \backslash F_{1}^{\mathbf{R}}$ by Theorem 6 .

The image by $i^{\mathbf{R}}$ of the canonical (I)-regular stratification of $V \backslash F_{1}$ is $\mathcal{S}_{1}^{\mathbf{R}}$.

The step $i \geq 2$ :

* Test by means of Theorem 5 if $F_{i-1}^{\mathbf{R}}=\emptyset$.

$*$ If $F_{i-1}^{\mathbf{R}}=\emptyset, l=i-1$, and the filtration computation is completed.

* Otherwise, $F_{i}^{\mathbf{R}}$ is built by

$$
\begin{aligned}
& F_{i}^{\mathbf{R}}=\left[\left(F_{i-1, \text { sing }}\right) \cup\left[\overline{\bigcup_{C \in \mathcal{S}_{i-1}} I\left(C,\left(F_{i-1, \mathrm{reg}} \cap \bar{C}\right)_{\mathrm{reg}}\right)} \cap V\right]\right. \\
& \left.\cup\left[\bigcup_{C \in \mathcal{S}_{i-1}}\left(\bar{C} \cap \overline{\left(F_{i-1} \backslash \bar{C}\right)} \cap V\right)\right]\right]^{\mathbf{R}} \text {. }
\end{aligned}
$$

Then

$$
\begin{aligned}
F_{i}^{\mathbf{R}}=\left(F_{i-1}^{\mathbf{R}}\right)_{\operatorname{sing} 1} & \cup\left[\bigcup_{D \in \mathcal{S}_{i-1}^{\mathbf{R}}} I\left(D^{\mathbf{C}},\left(\left(\left(F_{i-1}^{\mathbf{R}}\right)_{\operatorname{reg} 1} \cap \bar{D}\right)_{\operatorname{reg} 1}\right)^{\mathbf{C}}\right)^{\mathbf{R}} \cap V^{\mathbf{R}}\right] \\
\cup & {\left[\bigcup_{D \in \mathcal{S}_{i-1}^{\mathbf{R}}}\left(\bar{D} \cap \overline{\left(F_{i-1}^{\mathbf{R}} \backslash \bar{D}\right)} \cap V^{\mathbf{R}}\right)\right] . }
\end{aligned}
$$

The computation of $F_{i}^{\mathbf{R}}$ requires the same construction as the real semialgebraic case (proof of Theorem 8).

* Computation of the set, $\mathcal{T}_{i}^{\mathbf{R}}$, of all connected components of $F_{i-1}^{\mathbf{R}} \backslash F_{i}^{\mathbf{R}}$ by Theorem 6. 
The image by $i^{\mathbf{R}}$ of the canonical (I)-regular stratification of $V \backslash F_{i}$ is given by $\mathcal{S}_{i}^{\mathbf{R}}=$ $\mathcal{S}_{i-1}^{\mathbf{R}} \cup \mathcal{T}_{i}^{\mathbf{R}}$.

* The image by $i^{\mathbf{R}}$ of the canonical (I)-regular stratification of $V, \mathcal{S}^{\mathbf{R}}$, is $\mathcal{S}_{l}^{\mathbf{R}}$.

As in the real semialgebraic case, the sequential complexity of this algorithm is

$$
\left(\left(D^{n^{O(1)}}\right)^{\cdots{ }^{l \text { times } \cdots}}\right)^{n^{O(1)}}=D^{n^{0(l)}}
$$

It is doubly exponential in the depth of the associated filtration.

Thus, the following result is proved:

Theorem 11. Let (I) be an admissible stratifying condition over $\mathbf{R}[i]$.

Let $V$ be a constructible set of $\mathbf{C}^{n}$ defined by its image by the identification of $\mathbf{C}^{n}$ to $\mathbf{R}^{2 n}$ by the map $\left(z_{i}\right)_{i=1, \ldots, n} \mapsto\left(\operatorname{Re} z_{i}, \operatorname{Im} z_{i}\right)_{i=1, \ldots, n}$, by means of a quantifier-free formula of $\mathbf{R}$ of degree at most $D(D \geq n)$.

Let l be the depth of the filtration associated to the canonical (I)-regular stratification of $V$.

Then, there exists a well-parallelizable arithmetic network with a sequential complexity $D^{n^{o(l)}}$ which constructs quantifier-free formulas of $\mathbf{R}$ which define the image by the identification of $\mathbf{C}^{n}$ to $\mathbf{R}^{2 n}$ of each stratum of the canonical (I)-regular stratification of $V$ and also of each element of the associated filtration.

The proof provides the expected algorithm.

\subsection{Admissibility Over $\mathbf{R}[i]$ of the Whitney Conditions}

In order to prove the admissibility of the Whitney conditions over $\mathbf{R}[i]$, the same constructions as the real case are used here.

Proposition 6. The Whitney conditions $(a)$ and $(b)$ are both admissible stratifying conditions over $\mathbf{R}[i]$.

Proof. Taking the proof of Proposition 4 again is enough in order to get a proof of this proposition. Only small changes are necessary.

(1) The notations of the proof of Theorem 11 are used here again. Let $A$ be a constructible set of $\mathbf{C}^{n}$. Let $z$ be a point $C^{1}$-smooth of $A$. Then, $(\bar{A})^{\mathbf{R}}=\overline{A^{\mathbf{R}}}$ and $\left(T_{z}^{A}\right)^{\mathbf{R}}=T_{z}^{A} \mathbf{R}$. Those two properties are required during the proof.

(2) Let $k$ be an integer between 0 and $n$. The Grassmannian $\mathbf{G}_{n, k}(\mathbf{C})$ can be considered as a subset of $\mathbf{G}_{2 n, 2 k}(\mathbf{R})$ due to $i^{\mathbf{R}}$. According to Lemma 4, the Grassmannian $\mathbf{G}_{2 n, 2 k}(\mathbf{R})$ is represented by the subset of $\mathbf{M}_{2 n, 2 n}(\mathbf{R}) \simeq \mathbf{R}^{4 n^{2}}$ defined by

$$
\mathbf{G}_{2 n, 2 k}(\mathbf{R})=\left\{A \in \mathbf{M}_{2 n, 2 n}(\mathbf{R}): A={ }^{t} A, A^{2}=A, \operatorname{trace}(A)=2 k\right\} .
$$


A vector space of $\mathbf{R}^{2 n}$ is represented by the matrix of its orthogonal projection in the canonical basis of $\mathbf{R}^{2 n}$.

Let $\sigma$ be the matrix in the canonical basis of $\mathbf{R}^{2 n}$ of the linear map

$$
\begin{aligned}
\mathbf{R}^{2 n} & \longrightarrow \mathbf{R}^{2 n} \\
\left(x_{1}, y_{1}, \ldots, x_{i}, y_{i}, \ldots, x_{n}, y_{n}\right) & \longmapsto\left(-y_{1}, x_{1}, \ldots,-y_{i}, x_{i}, \ldots,-y_{n}, x_{n}\right) .
\end{aligned}
$$

The Grassmannian $\mathbf{G}_{n, k}(\mathbf{C})$ is represented by the following algebraic subset of $\mathbf{M}_{2 n, 2 n}(\mathbf{R})$ :

$$
\mathbf{G}_{n, k}(\mathbf{C})^{\mathbf{R}}=\left\{A \in \mathbf{M}_{2 n, 2 n}(\mathbf{R}): A={ }^{t} A, A^{2}=A, \operatorname{trace}(A)=2 k, \sigma A=A \sigma\right\} .
$$

Thus, the set $\left(\bigcup_{k=0}^{n} \mathbf{G}_{n, k}(\mathbf{C})\right)$ is represented by the set of all the matrices, $A$, of $\mathbf{M}_{2 n, 2 n}(\mathbf{R})$ such that $A={ }^{t} A$ and $A^{2}=A$ and $\sigma A=A \sigma$.

This representation of Grassmannians is used during the translation of the Whitney conditions into formulas of $\mathbf{R}$. After the translations into formulas of $\mathbf{R}$, the criterion (Lemma 6) leads to the admissibility over $\mathbf{R}[i]$.

The admissibility over $\mathbf{R}[i]$ of the Whitney conditions allows us to construct algorithms of stratification computation using Theorem 11.

Theorem 12. Let $V$ be a constructible set of $\mathbf{C}^{n}$ defined by its image by the identification of $\mathbf{C}^{n}$ to $\mathbf{R}^{2 n}$ by the map $\left(z_{i}\right)_{i=1, \ldots, n} \mapsto\left(\operatorname{Re} z_{i}, \operatorname{Im} z_{i}\right)_{i=1, \ldots, n}$ by means of a quantifierfree formula of $\mathbf{R}$ of degree at most $D(D \geq n)$. Let $l_{a}$ (resp., $\left.l_{b}\right)$ be the depth of the filtration associated to the canonical Whitney (a)-regular (resp., Whitney (b)-regular) stratification of $V$.

Then, there exists two well-parallelizable arithmetic networks such that:

(i) one of them constructs, with a sequential complexity $D^{n^{O(l a)}}$, quantifier-free formulas of $\mathbf{R}$ which define the images by the identification of $\mathbf{C}^{n}$ to $\mathbf{R}^{2 n}$ of each stratum of the canonical Whitney (a)-regular $C^{k}$-stratification of $V$ and also of each element of the associated filtration; and

(ii) the other constructs, with a sequential complexity $D^{n^{O\left(l_{b}\right)}}$, quantifier-free formulas of $\mathbf{R}$ which define the images by the identification of $\mathbf{C}^{n}$ to $\mathbf{R}^{2 n}$ of each stratum of the canonical Whitney (b)-regular $C^{k}$-stratification of $V$ and also of each element of the associated filtration.

The proof provides the expected algorithm.

\section{References}

[B1] K. Bekka. C-régularité et trivialité topologique, Warwick Singularity Theory Symposium, 1989, pp. 42-62, 1991.

[B2] K. Bekka. Regular quasi-homogeneous stratification, Séminaire Hawaii Marseille. Travaux en Cours. To appear.

[BCR] J. Bochnak, M. Coste, and M. F. Roy. Géometrie Algébrique Réelle, Springer-Verlag, New York, 1987.

[C] J. Canny. The Complexity of Robot Motion Planning, MIT Press, Cambridge, MA, 1989. 
[CGV] J. Canny, D. Grigor'ev, and N.Vorobjov. Finding connected components of a semialgebraic set in subexponential time, Springer AAECC J., 2 (1992), 217-238.

[CG] A. Chistov and D. Grigoryev. Subexponential time solving systems of algebraic equations II, manuscript, L.O.M.I. preprints E.10.83, Steklov Institute of Leningrad, 1983.

[DH] J. H. Davenport and J. Heintz. Real quantifier elimination is doubly exponential, J. Symbolic Comput., 5 (1988), 29-35.

[GWdPL] C. G. Gibson, K. Wirthmuller, A. A. Du Plessis, and E. J. N. Looijenga. Topological Stability of Smooth Mappings, Lecture Notes, vol. 552, Springer-Verlag, New York, 1976.

[GHRSV] D. Grigor'ev, J. Heintz, M. F. Roy, P. Solerno, and N. Vorobjov. Comptage des composantes connexes d'un ensemble semi-algébrique en temps simplement exponentiel, C.R. Acad. Sci. Paris, 311 (1990), 879-882.

[GV] D. Grigor'ev and N. Vorobjov. Solving systems of polynomial inequalities in subexponential time, J. Symbolic Comput., 5 (1988), 37-64.

[HRS1] J. Heintz, M. F. Roy, and P. Solerno. Sur la complexité du principe de Tarski-Seidenberg, Bull. Math. Soc. France, 118 (1990), 101-126.

[HRS2] J. Heintz, M. F. Roy, and P. Solerno. Description of the connected components of a semialgebraic set in simply exponentional time, Manuscript, IRMAR, Université de Rennes, 1991.

[M] J. N. Mather. Stratifications and Mappings, Dynamical Systems, Academic Press, New York, 1973, pp. 195-232.

[PR] J. B. Poly and G. Raby. Fonction distance et singularités, Bull. Sci. Math., 2e série, 108 (1984), 187-195.

[R] J. Renegar. On the computational complexity and geometry of the first order theory of the reals, Parts I-III, J. Symbolic Comput., 13 (1992), 255-352.

[T] B. Teissier. Variétés polaires 2. Multiplicités polaires, sections planes, et conditions de Whitney, Actes de la conférence de géométrie algébrique à La Ràbida, 1981, Lecture Notes, vol. 961, Springer-Verlag, New York, 1982, pp. 314-491.

[Th] R. Thom. Ensembles et morphismes stratifiés, Bull. Amer. Math. Soc., 75 (1969), 240-284.

[Tr] D. Trotman. Geometric versions of Whitney regularity, Math. Proc. Cambridge Philos. Soc., (1976), 80, 99.

[vzG] J. Von Zur Gathen. Parallel arithmetic computations: A survey, Proc. 13th Symp. MFCS 1986, Lecture Notes in Computer Science, vol. 233, Springer-Verlag, Berlin, 1986, pp. 93-112.

[V] N. Vorobjov. Effective stratification of regular real algebraic varities, Real Algebraic Geometry, Proceedings, Rennes, 1991, Lecture Notes, vol. 1524, Springer-Verlag, New York, pp. 402-415.

Received February 15, 1995, and in revised form January 29, 1997 and May 6, 1997. 